\title{
Exploring nanoscale magnetism in advanced materials with polarized X-rays
}

\author{
Peter Fischer \\ Center for X-ray Optics \\ Lawrence Berkeley National Laboratory \\ 1 Cyclotron Rd \\ Berkeley, CA 94270 \\ U.S.A \\ e-mail address: PJFischer@lbl.gov
}




\begin{abstract}
Nanoscale magnetism is of paramount scientific interest and high technological relevance. To control magnetization on a nanoscale, both external magnetic fields and spin polarized currents, which generate a spin torque onto the local spin configuration, are being used. Novel ideas of manipulating the spins by electric fields or photons are emerging and benefit from advances in nanopreparation techniques of complex magnetic materials, such as multiferroics, ferromagnetic semiconductors, nanostructures, etc.

Advanced analytical tools are needed for their characterization. Polarized soft X-rays using x ray dichroism effects are used in a variety of spectroscopic and microscopic techniques capable of quantifying in an element, valence and sitesensitive way basic properties of ferro(i)- and antiferromagnetic systems, such as spin and orbital moments, nanoscale spin configurations and spin dynamics with sub-ns time resolution. Future X-ray sources, such as free electron lasers will provide an enormous increase in peak brilliance and open the fs time window to studies of magnetic materials. Thus fundamental magnetic time scales with nanometer spatial resolution can be addressed.
\end{abstract}

This review provides an overview and future opportunities of analytical tools using polarized X-rays by selected examples of current research with advanced magnetic materials. 


\section{KEYWORDS}

nanomagnetism, X-ray magnetic dichroism, X-ray spectroscopy, X-ray microscopy, spin dynamics 


\section{Introduction}

\subsection{Magnetism - key to modern nanotechnology}

An important breakthrough in understanding the phenomenon of magnetism occurred at the beginning of the $20^{\text {th }}$ century, when Pauli introduced the concept of the spin of the electron. It became clear that the spin which is the carrier of a magnetic moment, has the property of angular momentum and as such follows quantum mechanical principles [1],[2]. A direct experimental proof of the close connection between angular momentum and magnetism is the EinsteinDeHaas effect, which was first observed in 1915 [3]. The interaction of individual spins is described by Pauli's exchange interaction where the energetic ground state favors a certain spin configuration, either parallel or antiparallel. However, it also became clear that the long range dipolar interaction of these spins requires that magnetism has to involve the correlation of all spins in a system, which makes magnetism a very complex many-body problem. A solid theoretical description of magnetism which is able to take this fully into account, is still not available.

Despite this lack of fundamental understanding of e.g. the very origin of exchange interaction, magnetic materials and magnetic properties constitute the backbone of various current technologies. Magnets are not only in use for a long time e.g. in transformers, generators and electric motors but the orientation of a magnetic moment in an external magnetic field has been considered as a basic concept for particularly magnetic storage and sensors devices from its beginning in the second half of the $20^{\text {th }}$ century.

The quest to increase storage density in order to cope with the huge increase of data required an enormous decrease of relevant length scales, such as the bit size, which are now approaching the nanometer regime. Magnetic storage technology has thus become a topic of nanoscience.

The basic interactions in magnetic materials are the exchange, anisotropy and dipolar interactions. In general these interactions compete, therefore the energetically lowest spin configuration is not the single spin configuration, where all spins would align parallel, but the system splits up into various domains [4], where the magnetization points in different orientations in neighboring domains. A fundamental magnetic length scale is the magnetic

$$
l_{K}=\sqrt{A / K}
$$


exchange length $l_{K}$ which reflects the competition between exchange $A$ and anisotropy $K$ constants,

and both of them are specific to the magnetic material. Typical values for exchange lengths in common magnetic materials are in the sub-10nm regime.

Research of magnetism on the nanometer length scale is not only scientifically very attractive, since the quantum mechanical properties of the individual spins become significant, but new phenomena emerge from confinement and proximity[5], [6]. This has led to important fundamental discoveries, such as the Giant Magnetoresistance (GMR) effect [7], [8], which was awarded the Noble Prize in Physics in 2007. The transport properties, i.e. the electric resistance in a sandwich of two ferromagnetic thin films, which are separated by a non-magnetic layer, depend strongly on the relative orientation of the magnetization in both layers. The GMR effect was introduced into read head technology almost immediately after its discovery and has pushed the storage density significantly.

A completely new area of electronics, spintronics has emerged from these scientific achievements. There, in addition to the charge of the electrons, it is the spin itself, which is used as a new degree of freedom in numerous classes of advanced materials, including organic semiconductors, carbon based materials, multiferroics, oxides, or complex Heusler alloys to name but a few [9]- [28].

Scaling down the feature sizes was also made possible by the development of novel nanostructuring technologies, such as e-beam lithography, which can be considered as a topdown approach. There is also the reverse, bottom-up approach, such as Molecular Beam Epitaxy, which can be used to generate particular structures through a tailored assembling. While MBE is a time consuming process, there is increased interest to investigate the potential of having the system undergo a self assembly process to create magnetic nanostructures on a large scale [29][33].

As important as the confinement and proximity effect is the materials science aspect to nanoscale magnetism research. Sample fabrication methods have developed in a large variety capable of producing specimens of high elemental purity, excellent growth characteristics which 
yield smooth surfaces and interfaces, and tailoring elemental composition to achieve new functionalities and physical and chemical behavior. Typical examples are multiferroics [34] [40], which combine several ferroic properties, such as ferroelectricity and ferromagnetism, which could potentially lead to an electric field control of magnetism. Heusler [41]-[48] alloys in compositions and structures, which are not commonly found in nature, exhibit enhanced magnetic properties, such as large spin polarization and magnetic semiconductors could potentially provide a room temperature superconducting material, which would revolutionize the steadily increasing energy demand worldwide.

\subsection{Manipulating spins in modern magnetic materials}

The scientific approach to understand magnetism is to interact with the magnetization and study its response. The conventional way to gain access to magnetization is through external magnetic fields, which goes back to the observation by Oersted, that a current running through a wire can reverse the magnetization in a ferromagnetic sample. This mechanism is still in use e.g. for the writing process in a magnetic hard disk. The associated long ranging dipolar field, however, creates severe problems, such as crosstalk between neighboring bits, as the dimensions of the bit size approach the nanoscale.

Another possibility to manipulate spins on the nanoscale is the injection of spin polarized currents into ferromagnetic elements. The basic idea is that the spins of the electrons in an electric current can exert a torque onto a non-collinear spin configuration and finally reverse it. The effect of spin torque was predicted by Berger and Slonciewski a while ago [49]-[50], but has received significant attention recently [51]-[59]. With regard to scaling, this effect is advantageous and therefore an increasing scientific and technological interest has evolved in the recent past. Spin torque effects are one of the many applications in spin electronics, where in addition to the charge of the electrons one takes full advantage of its spin as a new degree of freedom. A technological example, which involves the manipulation of nanoscale magnetic structures are storage devices, such as the racetrack memory[60], [61], where domain walls acting as basic information units are pushed along a wire by short electric pulses. There are also concepts of logic elements being discussed that fully rely on domain wall manipulation [62]. Another very active research area is the magnetic vortex structure [63], [64], which occurs in confined geometries such as thin magnetic disks. In the center of such as disk is a singularity, the 
vortex core, and its binary character (up or down) is not only considered for technological purposes [65], [66] but also in connection with life science or medical applications [67].

Information in today's semiconductor electronic devices and data storage technologies is mainly transported and manipulated by charge currents. With aggressively advanced miniaturization, heat dissipation and power consumption become significant obstacles and alternative technologies that address these problems are hence required. It is projected that the energy demand from consumer electronics in 2030 based on today's technologies would require the equivalent of 230 nuclear power plants.

One promising candidate to replace existing charge-based technologies (CMOS) is based on using spin currents and accumulations [9] and therefore the concept of pure spin currents, which are not accompanied by a net charge current, has recently received increased attention [19].

From a fundamental point of view these pure spin currents provide direct insights into spindependent physics and are completely undisturbed by charge transport [68]-[70]. With regard to technological applications, they offer large advantages, such as reduced power dissipation, absence of Oersted stray fields and decoupling of spin and charge noise.

Pure spin currents can be generated through non-local electrical injection, optical injection, spin pumping from a precessing ferromagnet and spin Hall effect [71]. A deep insight into the spin dynamics of such non-equilibrium spin accumulations has not yet been achieved.

Going beyond, it has been demonstrated that even photons themselves, which carry angular momentum can be used to manipulate the magnetization on the nanoscale. Since laser pulses are the fastest man-made events, this suggests that the control of magnetism by light would enable the fastest possible switching of magnetization [72]. First experiments have shown that the fastest time to flip the spin is deep in the few fs regime [73]-[75]. Corresponding to the fundamental length scale as described above, a fundamental time scale can be derived from the strength of exchange interaction, which points into the few fs regime.

It is not surprising, that studies of fs spin dynamics have become a very hot topic in nanomagnetism research, but as of now there are just a few experiments and the overall understanding of the underlying principles is more or less in its infancy. Finally, ultrafast spin 
dynamics with nanometer spatial resolution will address the most challenging and fundamental questions in nanomagnetism.

To be able to provide a solid and fundamental understanding of magnetism, advanced analytical tools are mandatory. Polarized soft X-rays are among the most promising probes for this purpose. The soft X-ray regime lies between the extended ultraviolet and the hard $\mathrm{x}$-ray regime. It encompasses photon energies between about $200 \mathrm{eV}$ up to $2 \mathrm{keV}$, which correspond to a photon wavelength range between $0.6-6 \mathrm{~nm}$. The attractive feature of this region in terms of Xray spectroscopies is that it gives access to the strong dipole-permitted (core 2p) to (valence 3d) excitations in transition metals such as Fe, Co, Ni, but also to the (core 3d) to (valence 4f) transitions in rare earth materials. These two classes of materials are the prototypes of magnetic materials both in a fundamental as well as an applied context. Whereas the magnetism in 3d transition metals is due to the delocalized $3 \mathrm{~d}$ bands, the strongly localized $4 \mathrm{f}$ levels determine the magnetic properties of rare earths. Combining TM systems with RE materials gives rise to a rich field of interesting physics reflecting the interplay between localized and itinerant behavior. Furthermore, the short wavelength of soft X-rays in the nanometer regime is of great advantage compared to the optical regime in terms of diffraction limited imaging techniques of magnetic materials on the nanoscale. Finally, the current development of next generation x-ray FEL sources will provide fsec soft x-ray pulses at high brightness, which will give access to fundamental magnetic time scales.

In the following we review the basic properties of soft polarized X-rays for the characterization of magnetic material that ultimately should be capable of addressing the magnetic properties at fundamental length and time scales. Examples of their use to explore novel functionalities in multicomponent, tailored materials are given.

\section{Advanced analytical X-ray tools for magnetic materials}

The key feature in the analysis of advanced magnetic materials is elemental specificity, which allows tracking down the impact, that each component contributes to the magnetic behavior. The spectroscopic response serves as a direct fingerprint in multicomponent materials.

Imaging magnetic structures and the corresponding fast and ultrafast spin dynamics in novel and advanced magnetic materials is a very appealing analytical approach and therefore a manifold of powerful imaging techniques have been developed and flourished recently. One can 
categorize them according to the probes they are using, such as electrons, photons or scanning probe techniques.

To name a few of the prominent techniques, Spin Polarized Scanning Tunneling Microscopy (SP-STM) is spearheading spatial resolution, since it can provide static images with almost atomic resolution [76]-[82]. On the other hand time resolved Kerr microscopy using the magneto-optical Kerr effect provides images with a time resolution down to fs regime, however, with a (diffraction) limited spatial resolution in the sub-micrometer range only[83][84]. None of these techniques is able to distinguish the magnetic response from individual components in multicomponent materials, which, however, is of paramount interest in the continuing endeavor to explore material with novel functionalities.

\subsection{Magnetic X-ray spectroscopy}

Since the availability of synchrotron radiation as a high brilliant source of polarized X-rays the effect of X-ray magnetic circular dichroism (X-MCD) in core-level absorption, which detects basically the dependence of the X-ray absorption coefficient of circularly polarized radiation on the magnetization in a ferromagnetic species in the vicinity of an absorption edge, has become a powerful tool to investigate the magnetism of solids, surfaces, thin films, nanostructures, even down to single molecules[85].

Polarized X-rays are nowadays abundantly available at synchrotron laboratories. At bending magnet stations a circular degree of polarization up to about $60-80 \%$ can be obtained by viewing the radiation emitted off-orbit under an angle of $<1 \mathrm{mrad}$ at the cost of reduced photon intensity. At third generation storage rings dedicated insertion devices, such as helical undulators in the soft X-ray energy range are high-brilliant sources with outstanding polarization features. They allow tuning of the X-ray polarization characteristics by a mechanical movement of e.g. magnet arrays, which subsequently forces the electron into different paths upon transversal of the undulator. Thus not only the circular degree of photon polarization can be switched, but also linearly polarization can be obtained with its plane oriented into various directions. This opens the door for X-ray magnetic linear dichroism (X-MLD) studies in antiferromagnetic systems.

One way to detect X-ray absorption coefficients is to record the transmission through a sample by counting the incoming and transmitted numbers of photons; it follows an exponential 
law. Given the limited penetration depth of soft X-rays below $1 \mathrm{keV}$ in matter this provides an information depth of about $100 \mathrm{~nm}$.

The physical origin of X-MCD and its global characteristics can be explained within a simple two step model. In the first step the absorption of a right (left) handed photon yields a photoelectron undergoing a dipolar transition into an unoccupied electronic state above the Fermi level. Elemental specificity is inherently provided by the characteristic energy of the absorption edge. Due to orbital momentum conservation and spin-orbit interaction the photoelectron acquires a finite spin $\left\langle\sigma_{\mathrm{z}}>\right.$ and orbital $<\mathrm{l}_{\mathrm{z}}>$ polarization in the photon beam direction z. In particular for the transition from an initial $\mathrm{p}_{1 / 2}$ and $\mathrm{p}_{3 / 2}$ spin-orbit state into a d-like final state $<\sigma_{\mathrm{z}}>$ amounts to $-1 / 2$ and $+1 / 4$, respectively while $<\mathrm{l}_{\mathrm{z}}>=+3 / 4$ for both cases.

For very low photoelectron energies $(\mathrm{E}<20 \mathrm{eV})$ and itinerant final states the absorption coefficient is described by Fermi's golden rule, i.e., $\mu_{1}(E) \sim|M(E)|^{2} \cdot \rho_{l}(E)$, where $\rho_{1}(E)$ reflects the density of states of the unoccupied bands with a defined angular momentum l near the Fermi level. Due to exchange splitting in a ferromagnetic system the absorbing atom reveals a magnetic spin moment if the majority (minority) band final states are shifted below (above) the Fermi level. Thus a local magnetic spin moment is induced, given by the excess of spins at the Fermi energy.

The second step for X-MCD now considers the fact, that due to Pauli's exclusion principle the spin/orbital polarized photoelectrons created in the absorption process with a circularly polarized photon can be seen as a probe for the final-state spin/orbital polarization projected onto the photon k-vector. Applying an external magnetic field it is possible to adjust the sample's magnetization and to align the magnetic moments, i.e., the spins of the majority electrons are parallel or antiparallel to the $\mathrm{z}$ direction. Thus according to Fermi's golden rule an integration of the spectroscopic dichroic signal, which is related to the difference in spin-up and spin-down density of states, measures directly the local spin moment. A similar consideration holds for the orbital momentum.

Hence X-MCD spectroscopy offers the potential to address local magnetic moments separated into spin and orbital contributions by applying magneto-optical sum rules which were developed by Thole [86] and Carra [87] in the early 1990s shortly after the first observation of a strong X-MCD effect in the 3d elements, Fe, Co and Ni [88]. In particular the information that 
can be obtained on the orbital contribution to the local magnetic moments is crucial to understand e.g. the origin of magnetic anisotropies. Although the general applicability of the sum rules from a rigorous theoretical point of view might be questionable, at least for the itinerant transition metal systems, like Fe, Co, and Ni the experimentally obtained results are satisfying and agree with theoretical expectations.

While X-MCD spectroscopy allows studying ferromagnetic system, antiferromagnetic systems can be studied by the analogous effect of X-ray Magnetic Linear Dichroism (X-MLD). Historically, linear magnetic dichroism is older than circular magnetic dichroism. The transfer of magneto-optical techniques from the visible to the x-ray regime appeared for a long time impossible due to the difficulty of generating and manipulating polarized light in this wavelength regime. In 1986, it was realized that the linear polarization of synchrotron radiation generated in a bending magnet could be put to use for investigating magnetic phenomena, and the first studies of magnetic dichroism in the $\mathrm{x}$-ray regime were carried around this time using linear light polarization. At that time atomic calculations which agreed very well with $3 \mathrm{~d} \rightarrow 4 \mathrm{f} \mathrm{X-ray}$ absorption spectra for the localized ground states of rare earth predicted strongly different $3 d$ absorption spectra for the electric field of the radiation being either parallel or perpendicular to the magnetic moment of the rare earth [89] which was soon after experimentally verified by polarization-dependent studies of Tb-Fe garnet [90]. In the transition metals the $2 \mathrm{p} \rightarrow 3 \mathrm{~d}$ absorption appears somewhat weaker because of the the quenching of the ground-state orbital magnetic moment by crystal field effects. The first $3 d$ system for which linear dichroism was established was $\mathrm{Fe}_{2} \mathrm{O}_{3}$ [91].

The origin of the X-MLD effect is a nonspherical distortion of the atomic charge induced by the spin-orbit interaction and therefore the X-ray absorption of linear polarized light depends on the orientation of the electric field vector of the photons and the magnetic axis in the system, which exists for both ferro- and antiferromagnets. However, the size of X-MLD is in general much weaker than the corresponding X-MCD effect. It is important to recall the following two relationships for the intensities of the X-MCD and X-MLD effects [92], resp.

$$
\begin{aligned}
& I_{X-M C D} \propto P_{\text {circ }} \vec{m} \cdot \vec{L} \propto P_{\text {circ }}\langle\vec{m}\rangle \cos \theta \\
& I_{X-M L D} \propto P_{\text {lin }}|\vec{m} \cdot \vec{E}| \propto P_{\text {circ }}\left\langle\vec{m}^{2}\right\rangle \cos ^{2} \theta
\end{aligned}
$$


The intensities for both X-MCD and X-MLD scale with the degree of polarization, circular $P_{\text {circ }}$ and linear $P_{\text {lin }}$, resp. While the X-MCD effect depends on the projection of the magnetic moment $\vec{m}$ onto the photon angular momentum $\vec{L}$, the X-MLD effect scales with the expectation value of the square of the magnetic moment $\left\langle\vec{m}^{2}\right\rangle$. A schematic in Fig. 1 shows the different experimental configurations for X-MCD and X-MLD. Very recently, advances in the instrumentation of X-MLD experiments have clearly shown the strong angular dependence of the $\mathrm{X}-\mathrm{MLD}$ on the relative orientation of polarization, magnetic moments, and crystallographic axes, which indicated the need to revisit previous interpretation of interface coupling phenomena based on X-MLD experiments [93].

\subsection{Magnetic X-ray microscopy}

Despite the advantage of inherently better spatial resolution with $\mathrm{x}$-rays compared to optical microscopies, the lack of appropriate X-ray optics has prevented X-ray microscopy for nearly 100 years, although immediately after the discovery of X-rays by W.C. Roentgen in 1885 the short wavelengths of the X-rays have been used to determine e.g. crystal structures. In the mid 1980s it was realized that Fresnel zone plates (FZP), which are circular gratings with a radially increasing line density can be used as diffractive optics to build X-ray microscopes [94], [95] and the concurrent maturity of nanotechnological tools such as e-beam lithography has then enabled the fabrication of high quality X-ray optics, which are now readily available[96],[97] .

FZPs can be designed and customized for specific purposes and applications. Varying a few parameters, such as $\Delta \mathrm{r}$, which is the outermost ring diameter, $\mathrm{N}$, the number of zones, and $\lambda$, the photon wavelength at which the FZP is operating, one obtains a spatial resolution which is proportional to $\Delta \mathrm{r}$, a focal length, which is $\sim 4 \mathrm{~N}(\Delta \mathrm{r}) 2 / \lambda$ and a spectral bandwidth which is $\sim 1 / \mathrm{N}$ [98]. The most advanced FZPs for soft X-ray microscopy have achieved now a spatial resolution better than 10nm [99],[100] andcurrent developments seem to make the $5 \mathrm{~nm}$ spatial resolution regime become feasible in the foreseeable future.

The optical setup of the full-field soft X-ray microscope end station XM-1, located at the Advanced Light Source in Berkeley CA, where the x-ray microscopy data presented in this review have been obtained is shown in Fig. 2 and described in detail elsewhere [101]. 
The principle of this instrument follows that of an optical microscope. The main components are

- a light source, which is the bending magnet source at a third generation X-ray synchrotron such as the ALS

- a condenser part, which is the first FZP, the condenser zone plate (CZP), acting in combination with a small pinhole close to the sample as both monochromator and illuminating optic

- a high resolution objective lens, the micro zone plate (MZP) and

- a two dimensional photon detector, which is a commercially available CCD system.

Spatial resolution is largely set by $\Delta \mathrm{r}$ of the MZP. The range of photon energies, which is related to elemental specificity, is determined by the spectral resolution of the illuminating part, which for this instrument is dominated by the CZP-pinhole arrangement. Finally, time resolution is limited by the time structure of the X-ray source, therefore progress towards better time resolution requires $\mathrm{X}$-ray sources with shorter time structures.

For magnetic imaging [102], [103] X-ray magnetic circular dichroism (XMCD), i.e. the fact that the absorption of circularly polarized X-rays depends strongly on the relative orientation between the photon helicity and the photon propagation direction is utilized to provide a strong magnetic contrast[104]. Since the XMCD effect occurs predominantly in the vicinity of X-ray absorption edges, such as the spin-orbit coupled $\mathrm{L}_{2}$ and $\mathrm{L}_{3}$ edges, which reflect the element specific binding energies of inner core electrons, XMCD adds an inherent elemental sensitivity to this analytical tool. Large XMCD effects with values up to $25 \%$ occur e.g. for $3 \mathrm{~d}$ transition metals such as Fe, Co, $\mathrm{Ni}$ [88]. It is worth noting that besides the dominant absorption contrast, which is related to the imaginary part of the scattering amplitude, also a magnetic phase contrast, which is related to the real part of the scattering amplitude and is connected to the imaginary part via a Kramers-Kronig transformation, can also be used for magnetic imaging [105], [106]

The penetrability of soft X-rays through matter, described by the X-ray absorption length, is typically in the few 100nm regime. Therefore the transmission geometry in MTXM probes the volume of the sample with a thickness up to few 100nm, which, however, matches perfectly most of the magnetic systems of interest, such as thin films or multilayered structures. It is 
interesting to note that the penetration depth of electrons used in transmission electron microscopy [107]- [112] and photons used in soft X-ray microscopy are quite similar, which allows to utilize the complementarity of Lorentz-TEM and magnetic soft X-ray microscopy [113].

As a pure photon-in/photon-out based technique magnetic fields of in principle any strength and pointing into any arbitrary direction can be applied during the recording of X-ray images. At XM-1 typical magnetic fields up to 2-3kOe in perpendicular geometry and about 1-2kOe along the plane of the sample can be applied. Samples with both perpendicular and in-plane magnetisation [114] can thus be investigated. To image in-plane components the sample has to be tilted at about an axis perpendicular to the photon beam propagation, which is commonly chosen to be 60 degrees, thereby reducing the magnetic contrast in those images by a factor 2

Time resolution, i.e. the direct observation of spin dynamics benefits from the inherently pulsed structure of X-ray synchrotron storage rings. For example, at the Advanced Light Source in Berkeley CA electrons circulate in the storage ring at a typical energy of $1.9 \mathrm{GeV}$ in so-called bunches at a velocity close to the speed of light. The typical bunch length corresponds to about 70ps; therefore the emitted X-ray flashes have the same length. Setting up a stroboscopic pumpprobe scheme one can study fast processes such as spin dynamics on the sub-100ps time scale in nanoscale magnetic elements. It is important to note that the low photon intensity per bunch prevents single shot time resolved experiments and therefore only the perfectly repeatable part of spin dynamics processes can be studied so far.

Various soft X-ray microscopy techniques utilizing magnetic dichroism effects have been developed in the recent past to image magnetic materials. First magnetic images have been reported in 1993 using an X-ray photoemission electron microscope (X-PEEM) [115], where the secondary electrons generated in the primary X-ray absorption process are sent through a high resolution electron optics to generate an image of the magnetic structure. X-PEEM offers a good surface sensitivity given by the limited escape depth of electrons in solids to a few $\mathrm{nm}$.

There are two complementary microscopy techniques, which utilize the Fresnel zone plates as described above. Similar to the situation with transmission electron or optical microscopes there is a scanning transmission X-ray microscope (STXM) [116], where the FZP is used to focus the X-rays onto the specimen, which is then raster scanned to build an image. STXM has 
the advantage to provide good spectral resolution for micro-spectroscopic studies and is rather flexible in the choice of point detectors. On the other hand, the full field transmission soft X-ray microscope (TXM) uses the FZP as a high resolution X-ray objective lens [117] Recently, both STXM and full field TXM have shown similar spatial resolution depending on the degree of coherent illumination. Whereas STXM has some advantage as a micro-spectroscopic tool, the full field system can cover easily a larger field of view at highest spatial resolution [118]. State of the art full field microscopes at undulator sources have demonstrated recently high spectral resolution micro-spectroscopy capabilities [119].

Complementary to lens-based x-ray microscopies, lensless imaging techniques which can utilize XMCD for magnetic studies, are beingnow developed [120], [121]. Essentially, these techniques work in reciprocal space and record a diffraction pattern from the sample, which is back-transformed into real space via sophisticated phase retrieval algorithms or holographic methods.

Similar to X-ray spectroscopies using both X-MCD and X-MLD effects X-ray microscopies can be used to study ferromagnetic systems with X-MCD and antiferromagnetic system with XMLD[122], [123]. The latter has been realized with X-PEEM only so far, although in principle any X-ray microscopy technique should be capable of such studies.

In the following section we will demonstrate the strength of X-ray spectroscopic and microscopic techniques by selected examples from current research. The choice of examples should not be considered a complete representation of research topics, but rather the intent is to give the reader the ability to evaluate the strengths - and limitations - of X-ray based analytical techniques for studies of magnetic materials. It is the unique combination of element specificity, high sensitivity and accessibility of small length and fast time scales, which will be essential to understand the fundamental physical principles and to enable advances in technologies of the future, e.g. in spintronics [124],[125]. 


\section{Examples of X-ray based studies of modern magnetic materials}

\subsection{Magnetic thin films}

Thin magnetic films have attracted scientific research considerably, since they allow to study proximity effects, such as interface exchange coupling, which is both fundamentally important, but has also a tremendous impact to technological applications[5],[126]. Whereas ferromagnetic systems have found widespread applications in technologies, antiferromagnetic systems, although originating from the same fundamental mechanism, namely, the quantum mechanical exchange interaction, are far less understood. Recently, antiferromagnetically coupled multilayers with materials exhibiting a strong perpendicular anisotropy have seen a significant interest [127], [128]. In perpendicular anisotropy systems with an antiferromagnetic interlayer coupling, there is an interesting competition between the exchange interaction favoring the antiparallel alignment, whereas the dipolar and Zeeman interaction favor the parallel configuration. And while dipolar interactions would create lateral domains, the Zeeman and exchange coupling favors a uniform lateral magnetization. This competition between interactions of various range give rise to numerous stable magnetic configurations and reversal mechanism that offer rich physics [129].

As a prototype system we want to show results from a bilayer structure consisting of a perpendicular anisotropy [Pt/Co $]_{50}$ multilayer and a TbFe ferromagnetic alloy [130]. Both the Pt/Co multilayer and the TbFe layer exhibit a strong out-of-plane anisotropy. The break in symmetry at the Co-Pt interface gives rise to perpendicular magnetic anisotropy, and the thin $\mathrm{Pt}$ layers couple adjacent Co layers ferromagnetically. On the other hand, the TbFe alloy is ferromagnetic with the Tb moments oriented antiparallel to the Fe moments. The interfacial exchange coupling between the Pt/Co and the TbFe films is dominated by the ferromagnetic CoFe exchange interaction, i.e. the Co and Fe moments are parallel aligned. As the Tb sublattice moments dominate the total $\mathrm{Tb}_{30} \mathrm{Fe}_{70}$ magnetization, the interfacial exchange coupling leads to an antiparallel alignment of the TbFe layer relative to the PtCo multilayer in the absence of a magnetic field. By inserting another Pt layer between these two constituent, one can tailor the degree of exchange interaction by varying the thickness of the Pt layer.

Sample preparation was done by co-evaporation of the pure metals onto a $100 \mathrm{~nm}$ thin $\mathrm{Si}_{3} \mathrm{~N}_{4}$ membrane to allow for sufficient penetration of the soft X-rays in the transmission microscopy 
and spectroscopy measurements. The actual composition was chosen to be $[\mathrm{Pt} 0.75 \mathrm{~nm} / \mathrm{Co}$ 0.25nm]50/Tb ${ }_{30} \mathrm{Fe}_{70}$ 25nm/ Pt 5nm (see Fig. 3 c)).

XMCD spectroscopy and microscopy are able to investigate the complexbehavior of these coupled system with elemental, i.e. layer sensitivity by choosing either the $\mathrm{Fe} \mathrm{L}_{3}$ edge at $707 \mathrm{eV}$ or the $\mathrm{Co}_{3}$ edge at $778 \mathrm{eV}$, respectively.

Both analyses were performed on the same sample in transmission geometry, where the transmitted photon intensity is proportional to the projection of the magnetization onto the photon propagation direction.

Fig. 3 shows the X-ray absorption for both circular polarizations and the XMCD spectra in an applied field of 7kOe, where the sample is magnetically fully saturated, as has been verified by magnetic hysteresis loop measurements [130]. The displayed photon energy spectrum covers the Fe and the Co L absorption edges between 700-800eV. A strong XMCD signal is observed at both edges, however with the sign at corresponding edges pointing in opposite directions. This immediately verifies the antiparallel alignment of the Fe and the Co moment, which is expected for the parallel alignment of the Pt/Co stack and the TbFe layer magnetization. While the XMCD signal provides information on the average element specific magnetization, magnetic soft X-ray microscopy allows now to study in detail the microscopic behavior of the individual layers.

Fig. 4 displays two $\mathrm{x}$-ray images covering a field of view of $6 \mu \mathrm{m}$ at a spatial resolution of about 25nm. Recording the X-ray images at the Fe and Co $\mathrm{L}_{3}$ edge provides a direct image of the magnetic domain structure in the individual layers. The images were recorded in an external field of 3kOe, where domains are formed in both the PtCo and the TbFe layer with an average domain size of about 200nm. It is very impressive to see that X-ray microscopy reveals directly that the magnetic microstructure is completely identical in each of the layers; however, the magnetic contrast is reversed. This again demonstrates that the Fe and the Co moments of the magnetic domains are antiparallel aligned. This observation is a direct proof that the local Fe and Co moments align even on the microscopic scale antiparallel in each of the layers.

\subsection{Magnetic nanostructures}

Aside from proximity effects, which dominated the previous example, it is also the confinement, which leads to interesting effects in nanoscale magnetic materials. Confinement 
can be achieved in various ways and artificially nanostructuring is probably the most prominent one. Electron beam lithography is the technique of choice and has become one of the standard tools in the synthesis of nanomagnetic samples [131].

Most commonly permalloy which is a soft magnetic material, is chosen for such studies. It is a NiFe alloy with generically 20\% Fe and $80 \%$ Ni content. It shows a high magnetic permeability around 2000-3000,a low coercivity at the order of $100 \mathrm{~A} / \mathrm{m}$, almost zero magnetostriction and a significant anisotropic magnetoresistance. The magnetostatic energy of permalloy is usually two to three orders of magnitude larger than the anisotropy energy. In the search for high permeability materials, permalloy was discovered in 1914 at Bell Laboratories. Choosing proper composition and heat treatment permeabilities of more than 100,000 have been obtained. and has since found widespread applications in various technological areas. Lateral confinement of Permalloy, particular in thin films (and as a function of film thickness) creates a variety of symmetric configurations [132], such as Landau patterns, which can be readily calculated by micromagnetic simulations [133]. In the realm of the development of high-density and ultrafast magnetic data storage devices magnetic nanostructures and in particular domain walls [134]- [139] or magnetic vortex structures [140]- [150] are objects of intense experimental and theoretical [151]- [155] research and permalloy is widely used for these studies as material of choice. In essence, Permalloy is not only found e.g. in nearly every magnetic technological application, but it also serves very often as an initial material in fundamental magnetic research, which will be illustrated in the following.

Spin dynamics, i.e. the temporal development of the magnetization $\vec{M}$ is described by the Landau-Lifshitz-Gilbert equation (LLG) of motion [156], [157]

$$
\frac{d \vec{M}}{d t}=-\gamma\left[\vec{M} \times \vec{H}_{e f f}\right]+\frac{\alpha}{|M|}\left[\vec{M} \times \frac{d \vec{M}}{d t}\right]
$$

where the first term accounts for the precession of the magnetization in an external magnetic field $H_{\text {eff }}$ with $\gamma$ being the gyromagnetic ratio and the second term describes the relaxation and damping of the system with $\alpha$ being a damping constant depending strongly on the local geometry, anisotropy and morphology. Typical precession frequencies for micronsized elements are in the $\mathrm{GHz}$ regime, while relaxation times can extend into several ns. Studies of spin 
dynamics phenomena have attracted recent interest, particular when combined with short length scales. This is driven by both the demand from technologies, such as the demand for higher storage densities and minituarized magnetic sensors, but also by the quest to increase the speed at which e.g. information can be written. Scientifically, there are many fundamental questions, that need to be answered, such as

- is there an ultimate speed at which the magnetization can be reversed?

- are there other ways than applying external magnetic fields to reverse the spin configuration?

Although these question are only a few examples, it is obvious that they are intimately connected to specific and tailored properties of advanced magnetic materials which needs capabilities to analyse them properly. The search for magnetic materials with unprecedented properties is therefore one of the greatest challenges in nanomagnetism research today.

Time resolved studies of spin dynamics with soft X-ray microscopy take advantage of the inherent time structure of current synchrotron storage rings, however, the fact that the number of photons per electron bunch at current third generation synchrotrons is rather low, a stroboscopic pump-probe scheme has to be used[158]- [161]. To accumulate sufficient photons e.g. for a single image, typically $10^{8-9}$ pump-probe cycles are needed. As stated before, this means that only fully reproducible processes can be studied or in other words, only the fully reproducible part of the magnetization dynamics can be seen.

The results presented here, were obtained at the ALS operating in the so-called 2-bunch mode operation, where two electron bunches, each 70ps in width circulate at a $3 \mathrm{MHz}$ frequency, i.e. separated by 328ns. The clock signal of the synchrotron triggers a fast electronic pulsar, which launches pulses with a rise time of about 100ps into a waveguide structure. These pump pulses create either a local Oersted field pulse or, if the current is sent through the magnetic element exhibits a spin torque onto the magnetic microstructure They can be delayed relative to the X-ray probe pulse to follow the time development of the excited magnetic domain pattern. The advent of the X-ray pulse onto the sample is monitored by a fast Avalanche photo diode giving an accurate measure of the arrival time of the photons on the sample.

A typical example for imaging the fast spin dynamics of a domain wall is shown in Fig. 5 [162]. Microstructures of 20nm thick permalloy $\left(\mathrm{Ni}_{80} \mathrm{Fe}_{20}\right)$ were prepared on $100 \mathrm{~nm}$ thick $\mathrm{Si}_{3} \mathrm{~N}_{4}$ 
membranes by electron beam lithography, thermal evaporation, and lift-off processing. They were contacted by wave guides fabricated via a second lithography step and by dc-magnetron sputtering of $2 \mathrm{~nm} \mathrm{Al}$ and $20 \mathrm{~nm}$ Au. A magnetic DW was prepared in the infinity shaped Permalloy sample by applying an external magnetic field perpendicular to the direction of the coplanar waveguide. Once the magnetic field was released a DW was confined between the two openings in the infinity structure. Subsequently, short current pulses were sent through the coplanar waveguide (pump pulse) and synchronized to the X-ray flashes (probe pulse). Both a spin torque and a magnetic field were thus exciting the DW motion In Fig. 5 the reaction of the DW to the applied pulse is shown. The X-ray images taken at varying delay times between the pump and the probe pulse are shown as inserts. They are displayed as differential images normalized to the unexcited state. Therefore the white and black area denotes that the DW is moving up and down similar to an excited guitar string. The data points in Fig. 5 are the DW deflection as a function of the delay time superimposed to the pump profile between 1-2ns (gray line). It is evident, that the DW oscillations set in shortly after the onset of the pump pulse but extend for several ns after the excitation pulse length. A thorough analytical analysis of the DW oscillation allows both to derive essential parameters, such as the DW velocity, but moreover the experimental data could only be interpreted by taking into account a pinning potential which had to go beyond the harmonic order [162].

\subsection{Magnetic semiconductors}

The following paragraphs describe various approaches to design novel magnetic materials that exhibit functionalities, which are of particular interest to technological applications.

The first class are magnetic semiconductors. They are essentiallysemiconductor materials, which are integral components to most of our current technologies, but combine both ferromagnetism and semiconductor properties. In the realm of spintronics a magnetic semiconductor at ambient conditions would provide a way to not only control conduction through charge carriers, but also through the spin of the electrons. Dilute magnetic semiconductors (DMS) have recently risen to a major focus of magnetic semiconductor research 
[163] - [167]. These are based on traditional semiconductors, but are doped with transition metals instead of, or in addition to, electronically active elements. Among the current challenges for DMS are reproducible sample fabrication, since several studies on identical materials have shown diverging results and, most importantly, to increase their Curie temperatures into the room temperature regime, which is needed for a broad and practical applicability.

The first measurements of ferromagnetism in a semiconductor were done in Mn doped InAs and GaAs by Ohno et al. More recently a focus was given to doped oxide semiconductors such as Co doped $\mathrm{ZnO}$ [168]- [172] or $\mathrm{TiO}_{2}$ [173] - [174]. Other promising candidates for magnetic semiconductor applications due to its compatibility to the mainstream Si technology are Ge-Mn systems [175].

X-ray based analytical techniques, in particular X-ray absorption and X-MCD techniques are currently applied to any of these systems and provide a unique wealth of experimental data.In a very recent study in O-deficient $\mathrm{ZnO}$ :Cu films it was observed, that there are two distinct spectroscopic features of the $\mathrm{Cu}$ atoms, which could be associated with the degree of ordering, which are essential to observe ferromagnetism in these systems. The authors have interpreted their data by a microscopic indirect double-exchange model, where the alignment of localized $\mathrm{Cu}$ moments is mediated by vacancy orbitals. This constitutes a first direct evidence of ferromagnetism in O-deficient $\mathrm{ZnO}: \mathrm{Cu}$ at room temperature and points to the importance of $\mathrm{Cu}$ and oxygen vacancies to induce ferromagnetism in these systems. It is worth noting that it is the unambiguous fingerprint characteristics of X-ray absorption spectroscopy and XMCD as its magnetic counterpart obtained at both the $\mathrm{O}$ K-edge and the $\mathrm{Cu} \mathrm{L}$ edges, which has led to this conclusion [176].

\subsection{Multiferroic materials}

Multiferroics are materials which combine multiple order parameters, such as electric, magnetic and structural order, which result in a coupling of e.g. ferromagnetism and ferroelectricity [177]-[180]. Materials with such properties offer the possibility to control ferromagnetism with electric fields, which would have tremendous technological advantages. Replacing magnetic fields, which require comparatively large electric currents by electric fields in existing magnetic device applications would reduce significantly power consumption and would also enable enhanced miniaturization. Novel device paradigms could be envisaged, such 
as magnetoelectric storage elements, electrically tunable filter devices, and electric field manipulation of spintronics. The search for suited materials is therefore a very active research area. Unfortunately, single-phase multiferroics at ambient operating conditions are very rare, and therefore concepts to artificially synthesize composite phase materials have received increased scientific interest recently. Fueled by advances in thin film growth techniques, high-quality single-crystalline samples has led to the identifi cation of new types of multiferroics and has allowed to tailor their properties e.g. through strain engineering. One example are e.g. epitaxially grown $\mathrm{BiFeO} 3$ (BFO) thin filmsThey exhibit simultaneously both antiferromagnetic and ferroelectric properties at room temperature, which makes this material an interesting candidate for room-temperature applications. Structurally, BFO is a rhombohedrally distorted perovskite ferroelectric with large intrinsic polarization and eight possible polarization directions occurring along the pseudocubic $<111>$ body diagonals. It is also known to be a G-type, canted antiferromagnet, which means that the individual moments on each Fe ion are aligned parallel within a given pseudocubic plane and antiparallel between adjacent planes. What are possible mechanisms to control the ferromagnetism through electric fields? One possibility is to have the antiferromagnetic ferroelectric BFO in contact with a ferromagne. It is well known, that ferromagnetic layers in contact with an antiferromagnet exhibit the effect of exchange bias, which is a magnetic coupling occurring at the interface. In BFO, where such an antiferromagnet is the magnetoelectric multiferroic, in principle the interface coupling can be controlled and manipulated by applying an electric field to the ferroelectric. A recent study confirmed very impressively this approach by utilizing the full capabilities of soft X-ray microspectroscopy. Heterostructures of Au (2 nm)/CoFe (2.5-20 nm)/BFO (50-200nm)/SrRuO3 (SRO) (25-50 nm) were grown on SrTiO3 (STO) (001)-oriented substrates using pulsed laser deposition at $700 \mathrm{C}$ in 100 mtorr of oxygen. After cooling to room temperature in oxygen athmosphere the ferromagnetic CoFe films were grown in a vacuum sputtering system with a base pressure of 5×10-9 torr under an applied magnetic field of 2000e to induce a uniaxial anisotropy in the system. High resolution transmission electron microscopy showed a high quality of the $\mathrm{BFO} / \mathrm{CoFe}$ interfaces to exclude interdiffusion between these two components. To demonstrate the electrical switching of the ferromagnetic domains, the authors first switched a distinguished region of the BFO by scanning with the tip of a piezo force microscope before depositing the ferromagnetic CoFe layer. Imaging the magnetic contrast in the CoFe layer by element-specific 
X-ray photoemission electron microscopy at the Co $\mathrm{L}_{3}$ absorption edge revealed unambiguously that the ferromagnetic domain pattern follows exactly the imprinted ferroelectric domain in the BFO. The element specificity and spin sensitivity of X-ray microspectroscopy was the key role to unravel the two different coupling phenomena here, namely the internal, magnetoelectric coupling between antiferromagnetism and ferroelectricity in the $\mathrm{BiFeO}$ film and the exchange interactions at the interface between a ferromagnet (Co0.9Fe0.1) and the antiferromagnet.

An alternative approach to control the spin configuration in a ferromagnet by electric fields is to use a ferroelectric layer as a tunnel barrier between two ferromagnetic layers. This concept has been recently studied in a system, where a the ferroelectric BaTiO3 film was sandwiched between a magnetic Fe and a La0.67Sr0.33MnO3 layers. Theoretical calculations have predicted the possibility of significant changes in the interfacial magnetization and spin polarization in a ferromagnet in response to the ferroelectric polarization state across the interface. Using epitaxial synthesis, scanned probe imaging and high-resolution electron microscopy, the feasibility of this concepts was also reported recently. The experimental signature was the observation of a large tunnelling magnetoresistance at low temperatures, which is very sensitive to the polarization state of the ferroelectric [177].

\subsection{Heusler alloys}

Although Heusler alloys have been known since more than 100 years, when Fritz Heusler discovered that a mixture of $\mathrm{Cu}_{2} \mathrm{Mn}$ with various metals such as $\mathrm{Al}, \mathrm{Si}$, Sn or Sb exhibited ferromagnetism despite the fact that all constituents are non-magnetic, they have received significant interest in the mid 80s with the discovery of a half-metallicity in NiMnSb and PtMnSb which rendered these systems to be useful for magneto-optical storage due to their high Kerr rotation.

In 2003 a CoCrFeAl compound showed a large magnetoresistance effect and since then Heusler alloys have become one of the most promising materials for spintronic applications due their high spin polarization, which is essential for efficient spintronics devices[183], [184].

The intermetallic and multicomponent character of Heusler alloys is an intriguing argument for studies of Heusler alloys with X-ray spectroscopy techniques and it is not surprising that in the recent past an abundance of experimental data have been accumulated on Heusler alloys to determine e.g. the individual spin and orbital contribution for each element and to measure the 
electronic states and to compare with theoretical first principle calculations to name but a few scientific topics [185]-[192].

We want to illustrate the importance of X-ray based analytical techniques to understand the magnetic properties in such Heusler systems by two examples from current research. The unique property of half-metallic ferromagnetism, where the majority spin band exhibits a metallic character, while the minority-spin band has an energy gap at the Fermi level, is not only scientifically very interesting, but makes materials with such a property extremely attractive for spintronic applications, where a high spin polarization is mandatory. Therefore numerous efforts, both experimentally and theoretically is devoted to understand the origin of band gaps in halfmetallic ferromagnets and Heusler alloys are among those systems. Whereas spin resolved photoemission can probe the spin polarization fo the half-metal surface, the even more interesting interface region between for example the half-metal and a barrier layer in a magnetic tunnel junction cannot be addressed by this techniques. It has also been predicted, that the localization of magnetic moments in Heusler alloys is deeply connected to the band-gap of the minority-spin states. Probing the localization of magnetic moments in an element-specific way is therefore a key to a fundamental understanding. A recent experimental study, which combined X-MCD and X-MLD has evidenced significant difference in the localization of the Co moment when comparing the Heusler compounds Co2MnSi against Co2MnAl. Epitaxial layers of Co2MnSi and Co2MnAl were grown on Cr-buffered $\mathrm{MgO}(001)$ substrates by inductively coupled plasma assisted magnetron sputtering from composition-adjusted sputtering targets. Xray diffraction confirmed the expected crystal structures of the Heusler alloy thin films. XMCD and XMLD spectroscopy was then applied at the L absorption edges to probe the localized character of the Co and Mn interface moments in those half-metallic Heusler films. The XMCD spectra showed a multiplet fine structure at both the Co and the Mn edges in Co2MnSi and an enhanced XMLD effect. In the corresponding Co2MnAl system, only the Mn absorption edges showed this behavior, whereas the signal at the Co was largely reduced suggesting that the Co moments are less localized in Co2MnAl. These observations are fully consistent with the theoretically predicted larger minority spin-gap in the Co partial density of states for the former compound as obtained from first principle calculations[193], [194].

The second example addresses the topic of demagnetization dynamics in the Heusler alloy Co2MnSi, which was experimentally studied by combining the element-specifity of XMCD 
spectroscopy with ultrafast (ps) time resolution. The mechanisms, which determine demagnetization in ferromagnetic metals are still unclear. Spin-flip scattering of hot electrons is one of the possible scenarios, which can occur at very fast time scales (100 fsec), however empty minority states at the Fermi level are prerequisite for this process. The time scale at which the demagnetization can occur is therefore driven by the amount of spin minority phase space. This topic is similar to the one described above and therefore Heusler alloys are again the materials of choice for such studies. In this study, the response to a ultrafast laser excitation pulse of a $\mathrm{Ni}$ film and a Co2MnSi Heusler alloy was studied by XMCD, where the ultrafast time resolution was provided by a streak camera. There the transmitted $\mathrm{x}$-ray pulses were converted into electrons, which are then dispersed by applying a fast sweeping voltage. The electrons are recorded by a linear detector and the location of incidence on the detector can be related to the time structure. A time resolution of a few ps can thus be obtained [195]. The streak images were collected at the Mn L3 edge in the Heusler compound and the Ni L3 edge of the Ni metal. The demagnetization data were collected in transmission, which probes the bulk of the sample. Very interestingly, this study revealed that the demagnetization process in the Heusler alloy, which was measured to be 3.5ps was significantly slower than in a conventional ferromagnet such as $\mathrm{Ni}$, where a $<1 \mathrm{ps}$ demagnetization time has been observed [196]. Fig. 7 shows the time resolved demagnetization profile of the Heusler compound in comparison with the ferromagnetic Ni film [192]. The faster decay for the Heusler alloy is clearly visible. This unexpected behavior could be attributed to a minority states band gap in the Heusler alloy, which prevents the ultrafast Elliot-Yafet type scattering mechanism.

While these results are already impressive by itself and demonstrate nicely the unique capabilities of X-rays, they are just a glimpse of what ultrafast X-ray based techniques can provide at upcoming X-ray sources (see chapter 4 below) in the near future.

\subsection{Magnetic molecules}

In the final example of research of magnetic materials with X-ray based analyses, the current limitations in terms of sensitivity are demonstrated.

The ability to manipulate electron spins in organic molecular materials has triggered a conceptually exciting branch of spintronics, the so-called field of molecular spintronic [197] [199]. Magnetic molecules have several advantages which make them feasible candidates for 
nanoscale information technology. The weak spin-orbit and hyperfine interactions in organic molecules opens the possibility of preserving spin-coherence over times and distances much longer than in conventional metals or semiconductors. At low temperatures the relaxation of magnetic molecules is very slow, and therefore they will keep their magnetization over a long period of time. However, the characterization of magnetic molecules is a real challenge for any analytical tool in terms of sensitivity and the requirement to operate at low temperatures. Using X-ray absorption spectroscopy and X-MCD techniques valuable insights could be obtained recently with various magnetic molecules [200]. Fig. 6 shows the structure of a star-shaped molecule comprising a $\mathrm{Mn}_{4} \mathrm{O}_{6}$ core. X-ray absorption and X-MCD spectroscopy was performed at BL 4.0.2 at the Advance Light Source in Berkeley with the samples mounted into a cryostat equipped with a 6T superconducting magnet. The experiments were performed at a temperature of $5 \mathrm{~K}$ and in a field of $5 \mathrm{~T}$ around the Mn L edges around $640 \mathrm{eV}$. The XAS and X-MCD spectra are displayed in Fig. 6. The XAS spectra, which are sensitive to the chemical environment of the absorbing atom, clearly revealed a $\mathrm{Mn}^{2+}$ ionic valence state. The corresponding X-MCD spectra and their analysis within the sum rules revealed that this molecule exhibits at a field of 5T a spin moment of $17.15 \mu_{\mathrm{B}} /$ formula unit and an orbital moment of $0.36 \mu_{\mathrm{B}} /$ f.u. These data confirm the weak coupling between the central ion and the three outer ions, which reflects the general weak ferromagnetic coupling of this molecule [201]. 


\section{$4 \quad$ Future directions in magnetism research with X-rays}

\subsection{Approaching fundamental length and time scales in magnetism}

Research on magnetism has come a long way and maintains to be a very active area in solid state physics. Its attractiveness can be seen by the fact, that magnetism not only is one of the dominant physical mechanisms which has led the information technology to the level we enjoy today, but that there are still very fundamental questions unsolved, such as the origin of exchange interaction. To answer those questions, the fundamental length and time scales, which govern magnetism need to be explored. The future directions of magnetism research and progress in the understanding of this phenomenon are therefore intimately linked to the enhancements of the capabilities of experimental analytical tools and theoretical models, which can address these areas. While the fundamental length scale in the nanometer regime has been reached both experimentally and also almost technologically, the gap to fundamental magnetic time scales in the fs regime at nanometer spatial resolution is wide open.

\subsection{From optomagnetics to pure spin currents}

We would like to end this review by mentioning a few examples of current research, which explore new areas of magnetism research and will therefore require a thorough and fundamental understanding of spins on fundamental length and time scales.

- Is there a fundamental limit as to how fast one can change the direction of a spin of the electron?

Reversal by an external magnetic field, which is commonly done in magnetic storage devices, requires a torque acting on the spin, which generates a precessional motion. A recent experiment where the magnetic field pulse was generated by ultrarelativistic electrons in a particle accelerator [202] has shown that below 2 ps the magnetization becomes fractured, which results in a random switching behavior [203]. On the other hand, ultrafast optical pulses were used to demagnetize a ferromagnetic sample and this was found to occur in the subpicosecond regime [1]. Very recently, an all-optical reversal of the magnetization was observed by applying a single 40fs short circularly polarized laser onto a ferromagnetic 
sample [204]. The direction of the switching in this experiment was found to be determined only by the helicity of the light pulse. This process seems to occur not through precession, but rather via a strongly nonequilibrium state [205]. This effect is also currently explored in antiferromagnetic systems [206] and theoretical models are further being developed to support this explanation [207]. Technologically, this result could be of paramount importance to an ultrafast and efficient writing process in future magnetic storage devices. This experiment clearly tackles the fundamental time limit in magnetism; however, so far there is no experiment, which would be able to investigate this effect on a nanometer length scale.

- How fast can spin angular momentum be transferred to orbital momentum and vice versa and what are the basic mechanisms for these processes?

An intense fs optical laser pulse, which is absorbed by a metal pumps energy into electronic excitations of the system and it takes some time to relax into thermal equilibrium. The very basic process proceeds through various possible channels, but the timescale is ultimately determined by an energy transfer from the electronic system to the lattice of the specimen. The interaction with the spin system is thought to be a subsequent process triggered by spinorbit interactions and angular momentum conservation. However, there is also the possibility of a direct transfer from the electronic system into the spin system, which would then allow for a faster interaction. Whereas this is an exciting fundamental scientific question, this would also have a significant technological impact, since it would allow for switching magnetization much faster; and in turn this would increased writing speedfor information technologies. The sensitivity of X-MCD spectroscopy to the individual spin and orbital moments is the technique of choice to tackle this problem.

Although the time structure of current synchrotron sources seems to be too slow to study fs phenomena, the technique of slicing, which basically modulates with a fs optical laser source the electron bunches in the storage ring so that fs short X-ray pulses can be obtained, opens a possibility to access this interesting area of science [208], [209].

A recent pump-probe experiment [210], where a fs laser pulse was used to excite a the electronic states in a Ni film, which was then probed utilizing the fs capabilities at the slicing source at BESSY/Berlin shed light on this question [211] . The X-MCD signal was measured as a function of delay time and the results are shown in Fig. 7. For the first time, the 
quenching of spin angular momentum and its transfer to the lattice with a time constant of $120 \pm 70$ fs could be determined unambiguously. This experiment revealed an unexpected increase in valence-electron localization during the first $120 \mathrm{fs}$, possibly providing the driving force behind femtosecond spin-lattice relaxation and confirmed, within the timing accuracy of about 50fs a synchronous decrease of the X-MCD signal, which is a measure of the magnetic moments. These experiments are very challenging and suffer mostly from very low photon intensities for fs studies at current synchrotron sources, but they still provide an unambiguous insight into fs spin dynamics phenomena.

Whereas the future capabilities at $4^{\text {th }}$ generation $\mathrm{X}$-ray sources, such as X-ray free electron lasers (X-FEL) [212]- [217] or Energy Recovery Linacs [218], will provide fully coherent and ultrashort X-ray pulses with sufficient photons per pulse to allow for single shot experiments, an alternative route could be table-top sources[219], which have seen recent progress to expand into the EUV and soft X-ray photon regime by using higher harmonics. First experiments on ultrafast spin dynamics at e.g. the M edges of transition metals have been published [220] probably involving even higher order in the X-MCD signal [221].

- $\quad$ Are there new mechanisms, which could serve as guidance in the quest of exploring novel magnetic materials with advanced functionalities?

Elemental carbon is one of the most important constituents in nature and is abundantly available. A ferromagnetic order in carbon would therefore be highly advantageous, e.g. for application in biotechnology, although the origin of a magnetic order has been a mystery ever since. However, recently, a microspectroscopic study at room temperature utilizing the capability of a Scanning Transmission X-ray microscope to distinguish element-specific local magnetic moments at high spatial resolution of metal-free carbon which was irradiated by a proton beam allowed to unambiguously identify a ferromagnetic character and its origin could be traced back to the electron system of the carbon [222].

- Is it possible to disconnect the flow of spins from the charge of the electrons? Spin-polarized currents, which are intensely investigated in spintronics [223] - [228], allow manipulating through spin torque effects the spin configuration at the nanoscale. However, the 
idea of using pure spin currents only instead of flowing electrons would advance tremendously present technology particularly with regard to energy consumption. To achieve this one has to engage a mechanism which decouples the spin current from the electronic current. Spin-orbit coupling gives rise to spin-dependent scattering which couples charge and spin currents in conducting materials. Pure spin currents can therefore be generated e.g. via the spin Hall effect or through spin pumping [229]- [231]. Although these effects are currently under intense investigation in various materials, it remains open, whether and when technological applications based on pure spin currents will be developed.

X-ray based analytics will continue to play a significant role in the future in the study of nanomagnetism. Next generation instruments and sources are currently on their way, and more insight into fundamental processes of the scientifically exciting and technologically relevant phenomenon of magnetism can be expected. 


\section{ACKNOWLEDGEMENTS}

This work was supported by the Director, Office of Science, Office of Basic Energy Sciences, Materials Sciences and Engineering Division, of the U.S. Department of Energy under Contract No. DE-AC02-05-CH11231.

We want to thank the many colleagues for longstanding and fruitful collaborations, in particular M-Y. Im, W.L. Chao, E. Anderson, (CXRO), S.-Ch. Shin (KAIST Korea), D.-H. Kim (Chungbuk Natl. U, Korea), G. Meier, L. Bocklage, (U Hamburg Germany), S. Mangin, (U Nancy France), S.K. Kim, (Seoul Natl U Korea), A. Hoffmann, O. Mosendz, (ANL), C. Fadley (UC Davis), F. Hellman (UC Berkeley) and J. Kortright (MSD LBNL). A special thanks to the staff of CXRO and ALS for their continued support. 


\section{REFERENCES}

[1] W. Pauli, Z. Phys. 31, 765 (1925)

[2] G. E. Uhlenbeck and S. Goudsmit; Naturwissenschaften, 13, 953 (1925); Nature, Lond. 117, 264 (1926)

[3] A. Einstein and W. J. de Haas, Verhandl. Deut. Phys. Ges., 17, 152 (1915)

[4] A. Hubert, R. Schäfer, "Magnetic Domains: The analysis of Magnetic Microstructure” (Springer-Verlag, Berlin, 1998)

[5] S. D. Bader, Rev. Mod. Phys. 78, 1 (2006)

[6] M I Montero, K. Liu, O M Stoll, A Hoffmann, J J Åkerman, J I Martın, J L Vicent, S M Baker, T P Russell, C Leighton, J Nogues, I. K Schuller, J. Phys. D: Appl. Phys. 352398 (2002)

[7] P. Grünberg, R. Schreiber, Y. Pang, M.B. Brodsky and H. Sowers, Phys. Rev. Lett. 57(19) (1986) 2442

[8] M.N. Baibich, J.M. Broto, A. Fert, F. Nguyen Van Dau, F. Petroff, P. Eitenne, G. Creuzet, A. Friederich, and J. Chazelas, Phys. Rev. Lett. 61(21) (1988) 2472

[9] S.D. Bader and S.S.P. Parkin, Annu. Rev. Condens. Matter Phys. 1 3.1-3.18 (2010) doi:10.1146/annurev-conmatphys-070909-104123

[10] D. Ralph, R. Buhrman, in Concepts in Spintronics (ed. Maekawa, S.) (Oxford Univ. Press, 2006)

[11] C. Chappert, A. Fert, F. Nguyen Van Dau, Nature Materials 6, 813 (2007) 
[12] R. Ramesh, Nature Materials 9, 380 (2010)

[13] F. Kuemmeth, H.O.H. Churchill, P.K. Herring, C.M Marcus, Materials Today, 1318 (2010)

[14] F. Xiu, Y. Wang, J. Kim, A. Hong, J. Tang, A. P. Jacob, J. Zou, K.L. Wang, Nature Materials 9337 (2010)

[15] J.E. Moore, Nature 464194 (2010)

[16] S. P. Dash, S. Sharma, R. S. Patel, M.P. de Jong, R. Jansen, Nature 462, 491 (2009)

[17] C.H. Marrows, L.C. Chapon, S. Langridge, Materials Today, 1270 (2009)

[18] V. A. Dediu, L. E. Hueso, I. Bergenti, C. Taliani, Nature Materials 8, 707 (2009)

[19] C. Chappert, J.V. Kim, Nature Physics, 4837 (2008)

[20] S. Sanvito, Nature Materials 6, 803 (2007)

[21] N. Tombros, C. Jozsa, M. Popinciuc, H.T. Jonkman, B J. van Wees, Nature 448, 571 (2007)

[22] D. Houssameddine, U. Ebels,, B. Delaët, B. Rodmacq, I. Firastrau, F. Ponthenier, M. Brunet, C. Thirion, J.-P. Michel, L. Prejbeanu-Buda, M.-C. Cyrille, O. Redon, B. Dieny, Nature Materials 6, 447 (2007)

[23] D. D. Awschalom, M.E. Flatte, Nature Physics, 3153 (2007)

[24] Y.-W. Son, M. L. Cohen, S. G. Louie, Nature 444, 347 (2006)

[25] S.A. Wolf, D. Treger, A. Chtchelkanova, Mrs Bulletin, 31400 (2006)

[26] I. Zutic, M. Fuhrer, Nature Physics, 185 (2005) 
[27] Y. Ohno, D. K. Young, B. Beschoten, F. Matsukura, H. Ohno, D. D. Awschalom, Nature 402, 790 (1999)

[28] S. A. Wolf, D. D. Awschalom, R. A. Buhrman, J. M. Daughton, S. von Molnár, M. L. Roukes, A. Y. Chtchelkanova, D. M. Treger, Science, 2941488 (2001)

[29] O. Hellwig, J. K. Bosworth, E. Dobisz, D. Kercher, T. Hauet, G. Zeltzer, J. D. RisnerJamtgaard, D. Yaney, R. Ruiz, Appl. Phys. Lett. 96, 052511 (2010)

[30] B. D. Terris and T. Thomson, J. Phys. D: Appl. Phys. 38, R199 (2005).

[31] R. Ruiz, H. Kang, F. A. Detcheverry, E. Dobisz, D. S. Kercher, T. R. Albrecht, J. J. de Pablo, and P. F. Nealey, Science 321, 936 (2008)

[32] C. Ross, Annu. Rev. Mater. Res. 31, 203 (2001)

[33] H.-S. Kim, L. Bi, H. Paik, D.-J. Yang, Y. C. Park, G. F. Dionne, C.A. Ross, Nano Lett., $10597(2010)$

[34] J. Wang, J. B. Neaton, H. Zheng, V. Nagarajan, S. B. Ogale, B. Liu, D. Viehland, V. Vaithyanathan, D. G. Schlom, U. V. Waghmare, N. A. Spaldin, K. M. Rabe, M. Wuttig, R. Ramesh, Science 2991719 (2003)

[35] N. A. Spaldin, M. Fiebig, Science 309391 (2005)

[36] S.-W. Cheong, M. Mostovoy, Nature Materials 6, 13 (2007)

[37] D. Chiba, M. Sawicki, Y. Nishitani, Y. Nakatani, F. Matsukura, H. Ohno, Nature 455, $515(2008)$

[38] N. Hur, S. Park, P. A. Sharma, J. S. Ahn, S. Guha, S-W. Cheong, Nature 429, 392 (2004)

[39] W. Eerenstein, N. D. Mathur, J. F. Scott, Nature 442, 759 (2006) 
[40] L.W. Martin, Y.-H. Chu, R. Ramesh, Mater. Sci. Eng. R (2010), doi:10.1016/j.mser.2010.03.001

[41] B. Hillebrands and C. Felser (Guest Editors) J. Phys. D: Appl. Phys. 39 (2006)

[42] C. Felser and B. Hillebrands (Guest Editors) J. Phys. D: Appl. Phys. 40 (2007)

[43] C. Felser and B. Hillebrands (Guest Editors) J. Phys. D: Appl. Phys. 42080301 (2009)

[44] R. Kainuma, Y. Imano, W. Ito, Y. Sutou, H. Morito, S. Okamoto, O. Kitakami, K. Oikawa, A. Fujita, T. Kanomata, K. Ishida, Nature 439, 957 (2006)

[45] H. Koinuma, I. Takeuchi, Nature Mat. 3429 (2004)

[46] B. Balke, S. Ouardi, T. Graf, J. Barth, C.G.F. Blum, G. H. Fecher, A. Shkabko, A. Weidenkaff, C. Felser, Solid State Comm. 150529 (2010)

[47] K. Hamaya, H. Itoh, O. Nakatsuka, K. Ueda, K. Yamamoto, M. Itakura, T. Taniyama, T. Ono, M. Miyao, Phys. Rev. Lett. 102, 137204 (2009)

[48] R. Shan, H. Sukegawa, W. H. Wang, M. Kodzuka, T. Furubayashi, T. Ohkubo, S. Mitani, K. Inomata, K. Hono, Phys. Rev. Lett. 102, 246601 (2009)

[49] L. Berger, Phys. Rev. B 54, (1996) 9353

[50] J. Slonczewski, , J. Magn. Magn. Mater. 159 (1996) L1

[51] D.C. Ralph, M.D. Stiles, J. Magn. Magn. Mat 3201190 (2008)

[52] C. Burrowes, A.P. Mihai D. Ravelosona, J.-V. Kim, C. Chappert, L. Vila, A. Marty, Y. Samson, F. Garcia-Sanchez, L.D. Buda-Prejbeanu, I. Tudosa, E. E. Fullerton, J.P. Attane, Nature Phys. 617 (2010) 
[53] J.C. Sankey, Y.-T. Cui, J.Z. Sun, J.C. Slonczewski, R. A. Buhrman, D.C. Ralph, Nature Physics, 467 (2008)

[54] H. Kubota, A. Fukushima, K. Yakushiji, T. Nagahama, S. Yuasa, K. Ando, H. Maehara, Y. Nagamine, K. Tsunekawa, D.D. Djayaprawira, N. Watanabe, Y. Suzuki, Nature Physics, 4 37 (2008)

[55] M. Yamanouchi, D. Chiba, F. Matsukura, H. Ohno, Nature 428, 539 (2004)

[56] S. I. Kiselev, J. C. Sankey, I. N. Krivorotov, N. C. Emley, R. J. Schoelkopf, R. A. Buhrman, D. C. Ralph, Nature 425380 (2003)

[57] E. B. Myers, D. C. Ralph, J. A. Katine, R. N. Louie, R. A. Buhrman, Science 285 5429, 867 (1999)

[58] S. Mangin, D. Ravelosona, J.A. Katine, M.J. Carey, B.D. Terris, E.E. Fullerton, Nature Mater., 5210 (2006)

[59] S. Krause, L. Berbil-Bautista, G. Herzog, M. Bode, R. Wiesendanger, Science, 3171537 (2007)

[60] S. S. P. Parkin, US Patent 309,6,834,005 (2004)

[61] S.S. P. Parkin, M. Hayashi, L. Thomas, Science 320, 190 (2008)

[62] D. A. Allwood, Gang Xiong, M. D. Cooke, C. C. Faulkner, D. Atkinson, N. Vernier, R. P. Cowburn, Science 309, 1688 (2005)

[63] T. Shinjo, T. Okuno, R. Hassdorf, K. Shigeto, T. Ono, Science 289, 930 (2000)

[64] J. Miltat and A. Thiaville, Science 298555 (2002) 
[65] A. Drews, B. Krüger, G. Meier, S. Bohlens, L. Bocklage, T. Matsuyama, M. Bolte, Appl. Phys. Lett. 94, 062504 (2009)

[66] B. Pigeau, G. de Loubens, O. Klein, A. Riegler, F. Lochner, G. Schmidt, L. W. Molenkamp, V. S. Tiberkevich, A. N. Slavin, Appl Phys Lett 96, 132506 (2010

[67] D.-H. Kim, E. A. Rozhkova, I. V. Ulasov, S. D. Bader, T. Rajh, M. S. Lesniak, V. Novosad, Nature Materials 9, 165 (2010)

[68] G. Mihajlović, J. E. Pearson, M. A. Garcia, S. D. Bader, and A. Hoffmann, Phys. Rev. Lett. 103, 166601 (2009).

[69] O. Mosendz, J. E. Pearson, F. Y. Fradin, G. E. W. Bauer, S. D. Bader, and A. Hoffmann, Phys. Rev. Lett. 104, 046601 (2010).

[70] G. Mihajlović, J. E. Pearson, S. D. Bader, and A. Hoffmann, Phys. Rev. Lett. 104, 237202 (2010).

[71] A. Hoffmann, Phys. Status Solidi C 4, 4236 (2007).

[72] A.V. Kimel, A. Kirilyuk, P.A. Usachev, R.V. Pisarev, A.M. Balbashov, and Th. Rasing, Nature, 435, 655 (2005).

[73] C. D. Stanciu, F. Hansteen, A.V. Kimel, A. Tsukamoto, A. Itoh, A. Kirilyuk, Th. Rasing, Phys. Rev. Lett. 99 (2007)

[74] A. V. Kimel, A. Kirilyuk, Th. Rasing, Laser \& Photon. Rev. 1, No. 3, 275-287 (2007)

[75] K. Vahaplar, A. M. Kalashnikova, A.V. Kimel, D. Hinzke, U. Nowak, R. Chantrell, A. Tsukamoto, A. Itoh, A. Kirilyuk, Th. Rasing, PRL 103, 117201 (2009) 
[76] A. Wachowiak, J. Wiebe, M. Bode, O. Pietzsch, M. Morgenstern, R. Wiesendanger, Science 298, 577 (2002).

[77] M. Bode, Rep. Prog. Phys. 66 (2003) 523

[78] S. Loth, K. von Bergmann, M. Ternes, C.P. Lutz, A.J. Heinrich, “Quantized spin momentum transfer in atomic-scale magnetic devices”, Nature Physics (2010)

[79] A.F. Otte, M. Ternes, K. von Bergmann, S. Loth, H. Brune, C.P. Lutz, C.F. Hirjibehedin, A.J. Heinrich, Nature Physics 4, 847 (2008)

[80] C.F. Hirjibehedin, C.-Y. Lin, A.F. Otte, M. Ternes, C.P. Lutz, B.A. Jones, A.J. Heinrich, Science 317, 1199 (2007)

[81] C.F. Hirjibehedin, C.P. Lutz, A.J. Heinrich, Science 312, 1021 (2006)

[82] A.J. Heinrich, J.A. Gupta, C.P. Lutz, D.M. Eigler, Science 306, 466 (2004).

[83] J. P. Park, P. Eames, D. M. Engebretson, J. Berezovsky, P. A. Crowell, Phys. Rev. B 67, 020403(R) (2003).

[84] Y. Acremann, C. H. Back, M. Buess, O. Portmann, A. Vaterlaus, D. Pescia, H. Melchior, Science 290492 (2000)

[85] J. B. Kortright, D. D. Awschalom, J. Stöhr, S. D. Bader, Y. U. Idzerda, S. S. P. Parkin, I. K. Schuller, H. -C. Siegmann, J Mag Magn Mat, 2077 (1999)

[86] B.T. Thole, P. Carra, F. Sette, G. van der Laan, Phys. Rev. Lett. 681943 (1992)

[87] P. Carra, B.T. Thole, M. Altarelli, X. Wang, Phys. Rev. Lett., 70694 (1993)

[88] C. T. Chen, F. Sette, Y. Ma, and S. Modesti, Phys. Rev. B 42 (1990) 7262

[89] B. T. Thole, G. van der Laan, G. A. Sawatzky, Phys. Rev. Lett. 552086 (1985) 
[90] G. van der Laan, B.T. Thole, G.A. Sawatzky, J.B. Goedkoop, J.C. Fuggle, J.-M. Esteva, R. Karnatak, J. P. Remeika, H.A. Dabkowska, Phys. Rev. B 34, 6529 (1986)

[91] P. Kuiper, B. G. Searle, P. Rudolf, L. H. Tjeng, C. T. Chen, Phys. Rev. Lett. 70, 1549 (1993)

[92] J. Stoehr and H.C. Siegmann, „Magnetism”, Springer (2006)

[93] E. Arenholz, G. van der Laan, R.V. Chopdekar, Y. Suzuki, Phys Rev Lett 98, 197201 (2007)

[94] G. Schmahl, D. Rudolph, B. Niemann, O. Christ, Q. Rev Biophysics 13, 397 (1980)

[95] J. Kirz, C. Jacobsen, M. Howells, Q. Rev. Biophys. 2833 (1995)

[96] W. Chao, B.D. Harteneck, J.A. Liddle, E.H. Anderson, D. T. Attwood, Nature 435, 1210 (2005)

[97] J. Vila-Comamala, K. Jefimovs, J. Raabe, T. Pilvi, R.H. Fink, M. Senoner, A. Maaßdorf, M. Ritala, C. David, Ultramicroscopy 1091360 (2009)

[98] D.T. Attwood, "Soft X-rays and Extreme Ultraviolet Radiation: Principles and Applications”, Cambridge University Press (1999)

[99] W. Chao, J. Kim, S. Rekawa, P. Fischer, E.H. Anderson, Optics Express 17(20) 17669 (2009)

[100]W. Chao, et al. (2010) in preparation

[101] P. Fischer, T. Eimüller, G. Schütz, G. Denbeaux, A. Lucero, L. Johnson, D. Attwood, S. Tsunashima, M. Kumazawa, N. Takagi, M. Köhler, and G. Bayreuther, Rev. Sci. Instr. 72(5) (2001) 2322 
[102] P. Fischer, G. Schütz, G. Schmahl, P. Guttmann und D. Raasch, Z.f. Physik B 101 (1996) 313

[103] P. Fischer, T. Eimüller, G. Schütz, P. Guttmann, G. Schmahl, K. Prügl und G. Bayreuther, J. Phys. D: Appl. Physics 31(6) (1998) 649

[104] B.S. Kang, D.H. Kim, E. Anderson, P. Fischer, G. Cho, J. Appl. Phys. 98 (2005) 093907

[105] C. Chang, A. Sakdinawat, P. Fischer, E. Anderson, D. Attwood, Optics Letters 31(10) (2006) 1564

[106] A. Scherz, W. F. Schlotter, K. Chen, R. Rick, J. Stöhr, J. Lüning, I. McNulty, Ch. Günther, F. Radu, W. Eberhardt, O. Hellwig, S. Eisebitt, Phys. Rev. B 76, 214410 (2007)

[107]S . McVitie, J.N. Chapman, IEEE Trans Mag, 24, 1778 (1988)

[108]K.J. Kirk, J.N. Chapman, C.D.W. Wilkinson, Appl. Phys Lett. 71539 (1997)

[109]J. N. Chapman, I. R. McFadyen, S. McVitie, IEEE Trans Mag, 26, 1506 (1990)

[110]K. J. O’Shea, S. McVitie, J. N. Chapman, J. M. R. Weaver, Appl Phys Lett 93, 202505 (2008)

[111]M. Huber, J. Zweck, D. Weiss, Phys Rev B 77054407 (2008)

[112]C. Phatak, M.Tanase, A.K.Petford-Long, M.DeGraef, Ultramicroscopy 109(3) 264 (2009)

[113]M. Köhler, T. Schweinböck, T. Schmidt, J. Zweck, G. Bayreuther, P. Fischer, G. Schütz, T. Eimüller, P. Guttmann, G. Schmahl, J. Appl. Phys. 87, 6481 (2000)

[114] P. Fischer, T. Eimüller, G. Schütz, M. Köhler, G. Bayreuther, G. Denbeaux, D. Attwood, J. Appl. Phys. 89(11) (2001) 7159 
[115] J. Stoehr, Y. Wu, B.D. Hermsmeier, M.D. Samant, G.R. Harp, S. Koranda, D. Dunham, B.P. Tonner, Science 259658 (1993)

[116] J.B. Kortright, S. Kim, H. Ohldag, G. Meigs, T. Warwick, in: W. Meyer-Ilse, T. Warwick and D. Attwood (eds.), X-Ray Microscopy, American Institute of Physics, Melville, 50749 (2000)

[117]D.-H. Kim, P. Fischer, W. Chao, E. Anderson, M.-Y. Im, S.-C. Shin, and S.-B. Choe, J. Appl. Phys. 99, $08 \mathrm{H} 303$ (2006)

[118] P. Fischer, D.-H. Kim, W. Chao, J. A. Liddle, E. H. Anderson, and D. T. Attwood, Materials Today 9, 26 (2006)

[119] G. Schneider, P. Guttmann, S. Heim, S. Rehbein, D. Eichert, and B. Niemann, CP879, Synchrotron Radiation Instrumentation: Ninth International Conference AIP, ed Jae-Young Choi and Seungyu Rah (2007) 1291

[120] S. Eisebitt, J. Lüning, W. F. Schlotter, M. Lörgen, O. Hellwig, W. Eberhardt, J. Stöhr, Nature 432, 885 (2004)

[121] S. Streit-Nierobisch, D. Stickler, C. Gutt, L.-M. Stadler, H. Stillrich, C. Menk, R. Frömter, C. Tieg, O. Leupold, H. P. Oepen, G. Grübel, J Appl Physics 106, 083909 (2009)

[122] A. Scholl, J. Stoehr, J. Luening, J. W. Seo, J. Fompeyrine, H. Siegwart, J.-P. Locquet, F. Nolting, S. Anders, E. E. Fullerton, M. R. Scheinfein, H. A. Padmore, Science 2871014 (2000)

[123]F. Nolting, A. Scholl, J. Stöhr, J.W. Seo, J. Fompeyrine, H. Siegwart, J.-P. Locquet, S. Anders, J. Lüning, E.E. Fullerton, M.F. Toney, M.R. Scheinfein, and H.A. Padmore, Nature 405, 767 (2000) 
[124] S.A. Chambers, Y.K. Yoo, MRS BULLETIN, 28706 (2003)

[125]I. Zutic, J. Fabian, S. Das Sarma, Reviews Of Modern Physics, 76323 (2004)

[126]J. Nogues and I.K. Schuller, J. Magn. Magn. Mater. 192, 203 (1999)

[127]E.E. Fullerton, J.S. Jiang, C.H. Sowers, J.E. Pearson and S.D. Bader, Appl. Phys. Lett. 72, 380 (1998)

[128] O. Hellwig, A. Berger, J. B. Kortright and E.E. Fullerton, J. Magn. Magn. Mater. 319, 13 (2007)

[129] O. Hellwig, A. Berger and E.E. Fullerton, Phys. Rev. Lett, 91, 197203 (2003)

[130] S. Mangin, T. Hauet, P. Fischer, D. H. Kim, J. B. Kortright, K. Chesnel, E. Arenholz, and Eric E. Fullerton, Phys Rev B 78024424 (2008)

[131]L. Heyderman, F. Nolting, D. Backes, S: Czekaj, L. Lopez-Diaz, M. Kläui, U. Rüdiger, C.A.F. Vaz, J.A.C: Bland, R.J. Matelon, U.G. Volkmann, P. Fischer, Phys. Rev. B.73 (2006) 214429

[132] G. Meier, R. Eiselt, M. Bolte, M. Barthelmes, T. Eimüller, P. Fischer, Appl. Phys. Lett. 85(7) (2004) 1193

[133]M.J. Donahue ,D.G. Porter Interagency Report NISTIR 6376, National Institute of Standards and Technology, Gaithersburg, MD (1999)

[134]D. A. Allwood, Gang Xiong, M. D. Cooke, C. C. Faulkner, D. Atkinson, N. Vernier, R. P. Cowburn, Science 2962003 (2002)

[135] M. Hayashi, L.Thomas, R. Moriya, C. Rettner, S. S. P. Parkin, Science 320209 (2008) 
[136] M. Kläui, C. A. F. Vaz, J. A. C. Bland, W. Wernsdorfer, G. Faini, E. Cambril, L. J. Heyderman, F. Nolting, U. Rüdiger, Phys. Rev. Lett. 94, 106601 (2005)

[137] O. Boulle, J. Kimling, P. Warnicke, M. Kläui, U. Rüdiger, G. Malinowski, H. J. M.

Swagten, B. Koopmans, C. Ulysse, G. Faini, Phys. Rev. Lett. 101, 216601 (2008)

[138]G. Meier, M. Bolte, R. Eiselt, B. Krüger, D.-H. Kim., P. Fischer, Phys. Rev. Lett. 98, $187202(2007)$

[139] M. T. Bryan, P. W. Fry, P. J. Fischer, and D. A. Allwood, J. Appl. Phys. 103, 07 D909 (2008)

[140]K. Yamada, S. Kasai, Y. Nakatani, K. Kobayashi, H. Kohno, A. Thiaville, T. Ono Nature Mater. 6270 (2007)

[141]K. Buchanan, P.E. Roy, M. Grimsditch, F.Y. Fradin, K.Yu. Guslienko, S.D. Bader, V. Novosad, Nature Phys. 1172 (2005)

[142]B. Van Waeyenberge, et al., Nature 444, 461 (2006).

[143]H. Stoll, A. Puzic B. v. Waeyenberge, P. Fischer, J. Raabe, M. Buess, T. Haug, R. Höllinger, C.H. Back, D. Weiss, G. Denbeaux, Appl. Phys. Lett. 84(17) (2004) 3328

[144] Y. Acremann, J. P. Strachan, V. Chembrolu, S. D. Andrews, T. Tyliszczak, J. A. Katine, M. J. Carey, B. M. Clemens, H. C. Siegmann, J. Stoehr, Phys Rev Lett 96, 217202 (2006)

[145] S. Kasai, Y. Nakatani, K.. Kobayashi, H. Kohno T. Ono, Phys. Rev. Lett., 97, 107204 (2006)

[146] S. Kasai, P. Fischer, M-Y. Im, K. Yamada, Y. Nakatani, K. Kobayashi, H. Kohno, and T. Ono, Phys Rev Lett 101, 237203 (2008) 
[147] S.-H. Jun, J.-H. Shim, S.-K. Oh, S.-C. Yu, D.-H. Kim, B. Mesler, P. Fischer, Appl Phys Lett 95142509 (2009)

[148] B.L. Mesler, P. Fischer, W. Chao, E.H. Anderson, D.-H. Kim, J. Vac Sci Techn 25, 2598 (2007)

[149] K.W. Chou et al., Appl. Phys. Lett. 90, 202505 (2007)

[150]A. Vansteenkiste, K.W. Chou, M.Weigand, M. Curcic, V. Sackmann, H. Stoll, T.

Tyliszczak, G.Woltersdorf, C. H. Back, G. Schütz, B. VanWaeyenberge, Nature Physics, 5 332 (2009)

[151]K.Yu. Guslienko, B. A. Ivanov, V. Novosad, Y. Otani, H. Shima, K. Fukamichi, J. Appl. Phys. 91, 8037 (2002)

[152] K.Yu. Guslienko and V. Novosad, J. Appl. Phys. 96, 4451 (2004).

[153]K.-S. Lee et al, Phys Rev Lett 101267206 (2009)

[154] K.-S. Lee, S.-K. Kim, Y.-S. Yu, Y.-S. Choi, K. Y. Guslienko, H. Jung, and P. Fischer, Phys Rev Lett 101, 267206 (2008)

[155]K.-S. Lee and S.-K. Kim, Phys. Rev. B 78014405 (2008)

[156] L.D. Landau, E.M. Lifshitz, Phys. Z. Soviet Union 8, 153 (1935)

[157] T.L. Gilbert, IEEE Trans Mag, 403443 (2004)

[158] S.B. Choe, et al. Science 304, 420-422 (2004)

[159]J. Raabe et al., Phys Rev Lett 94217204 (2005)

[160]P. Fischer, Current Opinion in Solid State and Materials Science 7 (2003) 173 
[161] L. Bocklage, B. Krueger, R. Eiselt, M. Bolte, P. Fischer, G. Meier, Phys Rev B 78 180405(R) (2008)

[162] L. Bocklage, B. Krueger, P. Fischer, G. Meier, Phys Rev B 81, 054404 (2010)

[163] J. M. D. Coey, M. Venkatesan, C. B. Fitzgerald, Nature Mat 4173 (2003)

[164] T. Dietl, H. Ohno, F. Matsukura, J. Cibert, D. Ferrand, Science 2871019 (2000)

[165] S. R. Dunsiger, J. P. Carlo, T. Goko, G. Nieuwenhuys, T. Prokscha, A. Suter, E. Morenzoni, D. Chiba, Y. Nishitani, T. Tanikawa, F. Matsukura, H. Ohno, J. Ohe, S. Maekawa,Y. J. Uemura, Nature Materials 9, 299 (2010)

[166] H. Ohno, D. Chiba, F. Matsukura, T. Omiya, E. Abe, T. Dietl, Y. Ohno, K. Ohtani, Nature 408944 (2000)

[167] H. Ohno, Science 281951 (1998)

[168] V. Ney, S. Ye, T. Kammermeier, K. Ollefs, A. Ney, T.C. Kaspar, S.A. Chambers, F. Wilhelm, A. Rogalev, J. of Magn. Magn Materials 3221232 (2010)

[169] A. Ney, K. Ollefs, S. Ye, T. Kammermeier, V. Ney, T. C. Kaspar, S. A. Chambers, F. Wilhelm, A. Rogalev, Phys. Rev. Lett. 100, 157201 (2008)

[170] K. Potzger, A. Shalimov, Shengqiang Zhou, H. Schmidt, A. MuÌ`cklich, M. Helm, J. Fassbender, M. Liberati, E. Arenholz, Journal of Applied Physics 105, 123917 (2009)

[171] M. H. N. Assadi, Y. B. Zhang, S. Li, Applied Physics Letters 95, 072503 (2009)

[172] T. Tietze, M. Gacic, G. Schütz, G. Jakob, S. Brück, E. Goering, New Journal Of Physics, 10055009 (2008) 
[173] H. Toyosaki, T. Fukumura, Y. Yamada, M. Kawasaki, Appl. Phys. Lett. 86, 182503 (2005)

[174] Y. J. Lee, M. P. de Jong, R. Jansen, Appl. Phys. Lett. 96, 082506 (2010)

[175] S. Ahlers, P. R. Stone, N. Sircar, E. Arenholz, O. D. Dubon, D. Bougeard, Appl. Phys. Lett. 95, 151911 (2009);

[176] T. S. Herng, D.-C. Qi, T. Berlijn, J. B. Yi, K. S. Yang, Y. Dai, Y. P. Feng, I. Santoso, C. Sanchez-Hanke, X.Y. Gao, Andrew T. S. Wee, W. Ku, J. Ding, A. Rusydi, Phys Rev. Lett. 105207201 (2010)

[177] V. Garcia, M. Bibes, L. Bocher, S. Valencia, F. Kronast, A. Crassous, X. Moya,S. EnouzVedrenne, A. Gloter, D. Imhoff, C. Deranlot, N. D. Mathur, S. Fusil, K. Bouzehouane, A. Barthélémy, Science 327, 1106 (2010)

[178] M. Fiebig, Th. Lottermoser, D. Fröhlich, A. V. Goltsev, R. V. Pisarev, Nature 419, 818 (2002)

[179] N. Hur, S. Park, P.A. Sharma, J.S. Ahn, S. Guha, S.W. Cheong, Nature, 429392 (2004)

[180] T. Zhao, A. Scholl, F. Zavaliche, K. Lee, M. Barry, A. Doran, M. P. Cruz, Y. H. Chu, C. Ederer, N. A. Spaldin, R. R. Das, D. M. Kim, S. H. Baek, C. B. Eom, R. Ramesh, Nature Materials 5, 823 (2006)

[181] Y.-H. Chu, L.W. Martin, M.B. Holcomb, M. Gajek, S.-J. Han, Q. He, N. Balke, C.-H. Yang, D. Lee, W. Hu, Q. Zhan, P.-L. Yang, A. Fraile-Rodríguez, A. Scholl, S. X. Wang, R. Ramesh, Nature Materials 7478 (2008) 
[182] M. B. Holcomb, L. W. Martin, A. Scholl, Q. He, P. Yu, C.-H. Yang, S. Y. Yang, P.-A. Glans, M. Valvidares, M. Huijben, J. B. Kortright, J. Guo, Y.-H. Chu, R. Ramesh, Phys. Rev. B 81134406 (2010)

[183] T. Block, C. Felser, G. Jakob, J. Ensling, B. Mühling, P. Gütlich, R. J. Cava, Journal of Solid State Chemistry, 176646 (2003)

[184] H. J. Elmers, G. H. Fecher, D. Valdaitsev, S. A. Nepijko, A. Gloskovskii, G. Jakob, G. Schönhense, S. Wurmehl, T. Block, C. Felser, P.-C. Hsu, W.-L. Tsai, S. Cramm, Phys. Rev. B 67, $104412(2003)$

[185] P. Klaer, M. Kallmayer, C. G. F. Blum, T. Graf, J. Barth, B. Balke, G. H. Fecher, C. Felser, H. J. Elmers, Phys. Rev. B 80, 144405 (2009)

[186] R Y Umetsu, T Nakamura, K Kobayashi, R Kainuma, A Sakuma, K Fukamichi, K Ishida, J. Physics D-Applied Physics, 43105001 (2010)

[187] T. Kubota , K. Kodama, T. Nakamura, Y. Sakuraba, M. Oogane, K. Takanashi, Y. Ando, Appl. Phys. Lett. 95, 222503 (2009)

[188] M. Jourdan, E.A. Jorge, C. Herbort, M. Kallmayer, P. Klaer, H.-J. Elmers, Appl. Phys. Lett. 95, 172504 (2009)

[189] S. Roy, E. Blackburn, S. M. Valvidares, M. R. Fitzsimmons, S. C. Vogel, M. Khan, I. Dubenko, S. Stadler, N. Ali, S. K. Sinha, J. B. Kortright, Phys. Rev. B 79, 235127 (2009)

[190] J. Schmalhorst, D. Ebke, M. Meinert, A. Thomas, G. Reiss, E. Arenholz, J. Appl. Phys. 105, $053906(2009)$

[191] P. Sharma, A. Gupta, K.V. Rao, F.J. Owens, R. Sharma, R. Ahuja, J.M.O. Guillen, B. Johansson, G.A. Gehring, Nature Materials, 2673 (2003) 
[192] Y. P. Opachich, A. Comin, A. F. Bartelt, A. T. Young, A. Scholl, J. Feng, J. Schmalhorst, H. J. Shin, K. Engelhorn, S. H. Risbud, G. Reiss, H. A. Padmore, J. Phys.: Condens. Matter $22156003(2010)$

[193] N. D. Telling, P. S. Keatley, G. van der Laan, R. J. Hicken, E. Arenholz, Y. Sakuraba, M. Oogane, Y. Ando, K. Takanashi, A. Sakuma, T. Miyazaki, Physical Review B 78, 184438 (2008)

[194] J. Kubler, A.R. Williams, C.B. Sommers, Physical Review B, 281745 (1983)

[195] A.F. Bartelt, A. Comin, J. Feng, R. Nasiatka, T. Eimuller, B. Ludescher, G. Schutz, H.A. Padmore, A.T. Young and A. Scholl Appl. Phys. Lett. 90162503 (2007)

[196] E. Beaurepaire, J.-C. Merle, A. Daunois, J.-Y. Bigot, Phys Rev Lett 764250 (1996)

[197] A. R. Rocha, V. M. García-Suárez, S. W. Bailey, C. J. Lambert, J. Ferrer, S. Sanvito, Nature Materials 4, 335 (2005)

[198] M. Mannini, F. Pineider, P. Sainctavit, C. Danieli, E. Otero, C. Sciancalepore, A.M. Talarico, M.-A. Arrio, A. Cornia, D. Gatteschi, R. Sessoli, Nature Materials 8, 194 (2009)

[199] L. Bogani, W. Wernsdorfer, Nature Materials 7, 179 (2008)

[200] S. Khanra, K. Kuepper, T. Weyhermueller, M. Prinz, M. Raekers, S. Voget, A.V.

Postnikov, F.M. F. de Groot, S. J. George, M. Coldea, M. Neumann, P. Chaudhuri, Inorg. Chem. 47, 4605 (2008)

[201] M. Prinz, K. Kuepper, Ch. Taubitz, M. Raekers, S. Khanra, B. Biswas, T. Weyhermueller, M. Uhlarz, J. Wosnitza, J. Schnack, A.V. Postnikov, Ch. Schroeder, S. J. George, M. Neumann, P. Chaudhuri, Inorg. Chem. 49, 2093 (2010) 
[202] S. J. Gamble, M. H. Burkhardt, A. Kashuba, R. Allenspach, S. S. P. Parkin, H. C.

Siegmann, J. Stöhr, Phys. Rev. Lett. 102, 217201 (2009)

[203] I. Tudosa, C. Stamm, A. B. Kashuba, F. King, H. C. Siegmann, J. Stöhr, G. Ju, B. Lu, D. Weller, Nature 428, 831 (2004)

[204] C. D. Stanciu, F. Hansteen, A.V. Kimel, A. Kirilyuk, A. Tsukamoto, A. Itoh, Th. Rasing, Phys. Rev. Lett. 99, 047601 (2007)

[205] K. Vahaplar, A. M. Kalashnikova, A. V. Kimel, D. Hinzke, U. Nowak, R. Chantrell, A. Tsukamoto,. Itoh, A. Kirilyuk, Th. Rasing, Phys. Rev. Lett. 103, 117201 (2009)

[206] A.V. Kimel, B.A. Ivanov, R.V. Pisarev, P.A. Usachev, A. Kirilyuk, Th. Rasing, Nature Physics, 5727 (2009)

[207] J. H. Mentink, M. V. Tretyakov, A. Fasolino, M. I. Katsnelson, Th. Rasing, J. Phys.: Condens. Matter 22176001 (2010)

[208] R.W. Schoenlein, S. Chattopadhyay, H.H.W. Chong, T.E. Glover, P.A. Heimann, C.V. Shank, A.A. Zholents, M.S. Zolotorev, Science 2872237 (2000)

[209] A.A. Zholents, M.S. Zolotorev, Physical Review Letters, 76912 (1996)

[210] C. Stamm, T. Kachel, N. Pontius, R. Mitzner, T. Quast, K. Holldack, S. Khan, C.

Lupulescu, E. F. Aziz, M. Wietstruk, H. A. Duerr, W. Eberhardt, Nature Mat. 6740 (2007)

[211] S. Khan, K. Holldack, T. Kachel, R. Mitzner, T. Quast, Physical Review Letters, 97 074801 (2006)

[212] J.R. Schneider, Nuclear Instruments \& Methods In Physics Research Section AAccelerators Spectrometers Detectors And Associated Equipment, 39841 (1997) 
[213] K. Tiedtke, A. Azima, N. von Bargen, L. Bittner, S. Bonfigt, S. Dusterer, B. Faatz, U. Fruhling, M. Gensch, C. Gerth, N. Guerassimova, U. Hahn, T. Hans, M. Hesse, K. Honkavaar, U. Jastrow, P. Juranic, S. Kapitzki, B. Keitel, T. Kracht, M. Kuhlmann, W.B. Li, M. Martins, T. Nunez, E. Plonjes, H. Redlin, E.L. Saldin, E.A. Schneidmiller, J.R. Schneider, S. Schreiber, N. Stojanovic, F. Tavella, S. Toleikis, R. Treusch, H. Weigelt, M. Wellhofer, H. Wabnitz, M.V. Yurkov, J. Feldhaus, New Jour. Physics, 11023029 (2009)

[214] T. Shintake, 2007 IEEE Particle Accelerator Conference : 89, (2007)

[215] T. Shintake, H. Tanaka, T. Hara, T. Tanaka, K. Togawa, M. Yabashi, Y. Otake, Y. Asano, T. Fukui, T. Hasegawa, A. Higashiya, N. Hosoda, T. Inagaki, S. Inoue, Y. Kim, M. Kitamura, N. Kumagai, H. Maesaka, S. Matsui, M. Nagasono, T. Ohshima, T. Sakurai, K. Tamasaku, Y. Tanaka, T. Tanikawa, T. Togashi, S.K. Wu, H. Kitamura, T. Ishikawa, T. Asaka, T. Bizen, S. Goto, T. Hirono, M. Ishii, H. Kimura, T. Kobayashi, T. Masuda, T. Matsushita, X. Marechal, H. Ohashi, T. Ohata, K. Shirasawa, T. Takagi, S. Takahashi, M. Takeuchi, R. Tanaka, A. Yamashita, K. Yanagida, C. Zhang, Physical Review Special Topics-Accelerators And Beams, 12070701 (2009)

[216] J. Arthur, Rev Sci. Instruments, 731393 (2002)

[217] H. Winick, J. Electron Spectroscopy and Related Phenomena, 751 (1995)

[218] S.M. Gruner, D. Bilderback, I. Bazarov, K. Finkelstein, G. Krafft, L. Merminga, H. Padamsee, Q. Shen, C. Sinclair, M. Tigner, Rev. Scientific Instruments, 731402 (2002)

[219] M.M. Murnane, H.C. Kapteyn, M.D. Rosen, R.W. Falcone, Science, 251531 (1991) 
[220] C. La-O-Vorakiat, M. Siemens, M.M. Murnane, H. Kapteyn, S. Mathias, M.

Aeschlimann, P. Grychtol, R. Adam, C.M. Schneider, J.M. Shaw, H. Nembach, T.J. Silva, Physical Review Letters, 103257402 (2009)

[221] S. Valencia, A. Kleibert, A. Gaupp, J. Rusz, D. Legut, J. Bansmann, W. Gudat, P.M.

Oppeneer, Physical Review Letters, 104187401 (2010)

[222] H. Ohldag, T. Tyliszczak, R. Hoehne, D. Spemann, P. Esquinazi, M. Ungureanu, T. Butz, Phys Rev Lett 98, 187204 (2007)

[223] S. Maekawa, Nature Materials, 8777 (2009)

[224] S. Takahashi, S. Maekawa, Science and Technology of Advanced Materials, 9, 014105 (2009)

[225] T. Yang, T. Kimura, Y. Otani, Nature Physics, 4851 (2008)

[226] P. Sharma, Science 28307531 (2005)

[227] N.P. Stern, D.W. Steuerman, S. Mack, A.C. Gossard, D.D. Awschalom, Nature Physics, $4843(2008)$

[228] K. Uchida, S. Takahashi, K. Harii, J. Ieda, W. Koshibae, K. Ando, S. Maekawa, E. Saitoh, Nature 455, 778 (2008)

[229] O. Mosendz, G. Mihajlovic, J. E. Pearson, P. Fischer, M.-Y. Im, S. D. Bader, A. Hoffmann, Phys Rev B 80104439 (2009)

[230] O. Mosendz, J. E. Pearson, F.Y. Fradin, G. E.W. Bauer, S. D. Bader, A. Hoffmann, Phys Rev Lett 104, 046601 (2010) 
[231] O. Mosendz, G. Woltersdorf, B. Kardasz, B. Heinrich, C.H. Back, Physical Review B, 79 224412 (2009) 


\section{FIGURE CAPTIONS}

Figure 1: Schematic of the experimental geometry between photon polarization, magnetic field and photon propagation direction to detect (a) X-ray magnetic circular dichroism (X-MCD) (b) and X-ray magnetic linear dichroism (X-MLD).

Figure 2: Schematics of the optical setup of the full field soft X-ray microscope XM-1 located at beamline 6.1.2 at the Advanced Light source. Electrons in the storage ring emit X-rays in the bending magnet. The off-orbit emitted polarized X-rays are monochromatized by the condenser zone plate (CZP) - pinhole arrangement before they illuminate the magnetic sample. The microzone plate (MZP) downstream the sample projects a magnified image of the transmitted X-rays onto the 2dim CCD detector. From Ref. [118]

Figure 3: a) X-ray absorption spectra taken with left and right circularly polarized X-rays of a [Pt $0.75 \mathrm{~nm} / \mathrm{Co} 0.25 \mathrm{~nm}] 50 / \mathrm{Tb}_{30} \mathrm{Fe}_{70} 25 \mathrm{~nm} / \mathrm{Pt}$ nm layered film covering the Fe and Co L edges around 700-800eV.

b) XMCD recorded for this system showing in a layer resolved way the antiparallel coupling of the Fe and Co containing layers.

c) Scheme of the $[\mathrm{Pt} 0.75 \mathrm{~nm} / \mathrm{Co} 0.25 \mathrm{~nm}] 50 / \mathrm{Tb}_{30} \mathrm{Fe}_{70} 25 \mathrm{~nm} / \mathrm{Pt} 5 \mathrm{~nm}$ multilayered film. From Ref. [130].

Figure 4: Magnetic X-ray microscopy images of the [Pt $0.75 \mathrm{~nm} / \mathrm{Co} 0.25 \mathrm{~nm}] 50 / \mathrm{Tb}_{30} \mathrm{Fe}_{70}$ 25nm/ Pt 5nm multilayered film recorded at the Co $\mathrm{L}_{3}$ (left) and $\mathrm{Fe} \mathrm{L}_{3}$ (right) edge at a 
magnetic field of about 3kOe. The two images show the same domain pattern with reversed contrast demonstrating the antiparallel alignment of the Fe and Co moments and their direct coupling. From Ref. [130].

Figure 5: (a) Scheme of the infinity structure and its magnetization (arrows). (b) Corresponding differential X-ray images showing the static magnetic configuration with the domain wall spanning horizontally. (c) Time evolution of the vertical deflection of the domain wall. The gray curve depicts the applied current pulse. The dotted red curve represents a fit for the free oscillation of the domain wall upon excitation with a short current pulse. The images show differential X-ray images corresponding to the yellow square in (b) recorded at 200, 1800 and 2600 ps delay time between the electronic pump and the X-ray probe pulse. From Ref. [161]

Figure 6: (a), In-plane piezo force microscopy image showing the ferroelectric domain structure of a BFO film with a large (10 $\mu \mathrm{m}$, red square) and small (5 $\mu \mathrm{m}$, green square) electrically switched region. (b), Corresponding XMCD-PEEM image taken at the Co L-edge for a CoFe film grown on the written Pattern showing the direct match of the domain structures. (c) Rotation of the sample in reference to the incoming right-circularly polarized light enables allows to resolve the orientation of the magnetization in the CoFe layer. From Ref. [181]

Figure 7: (a), (b) XMCD transmission streaks at the Mn L3 edge (639.4 eV), with magnetization orientation anti-parallel and parallel to the polarization of incident $\mathrm{x}$-ray pulses. A difference streak is shown in (c). (d) Normalized Ni and Co2MnSi asymmetry signals and fits. From Ref. [192] 
Figure 8: Left: Molecular structure of a star-shaped molecule of $\mathrm{Mn}_{4}^{\mathrm{II}} \mathrm{O}_{6}$ core. Right: a) X-ray absorption spectra at the Mn L-edge for right and left circular polarized photons. b) Experimental and calculated XMCD spectra. From Ref. [200]

Figure 9: Femtosecond evolution of the electronic and magnetic structure in Ni.

a) Change of the XAS intensity with linearly polarized X-rays incident perpendicular to the sample surface versus pump-probe time delay b) Time-resolved XMCD signal with circularly polarized $\mathrm{X}$-rays incident at 60 relative to the sample surface versus pump-probe time delay. From Ref. [210] 


\section{FIGURES}

(a)

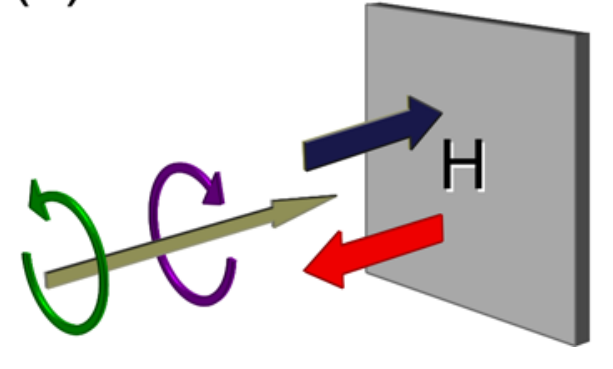

(b)

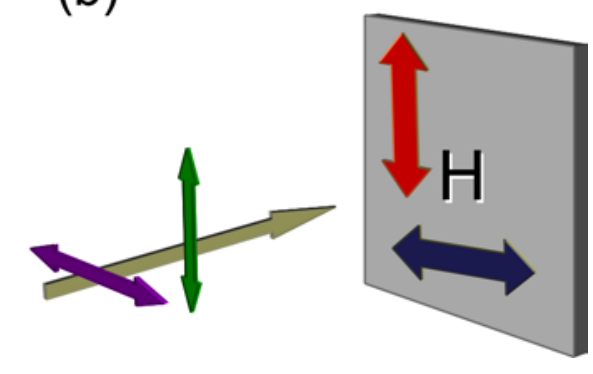

Figure 1 


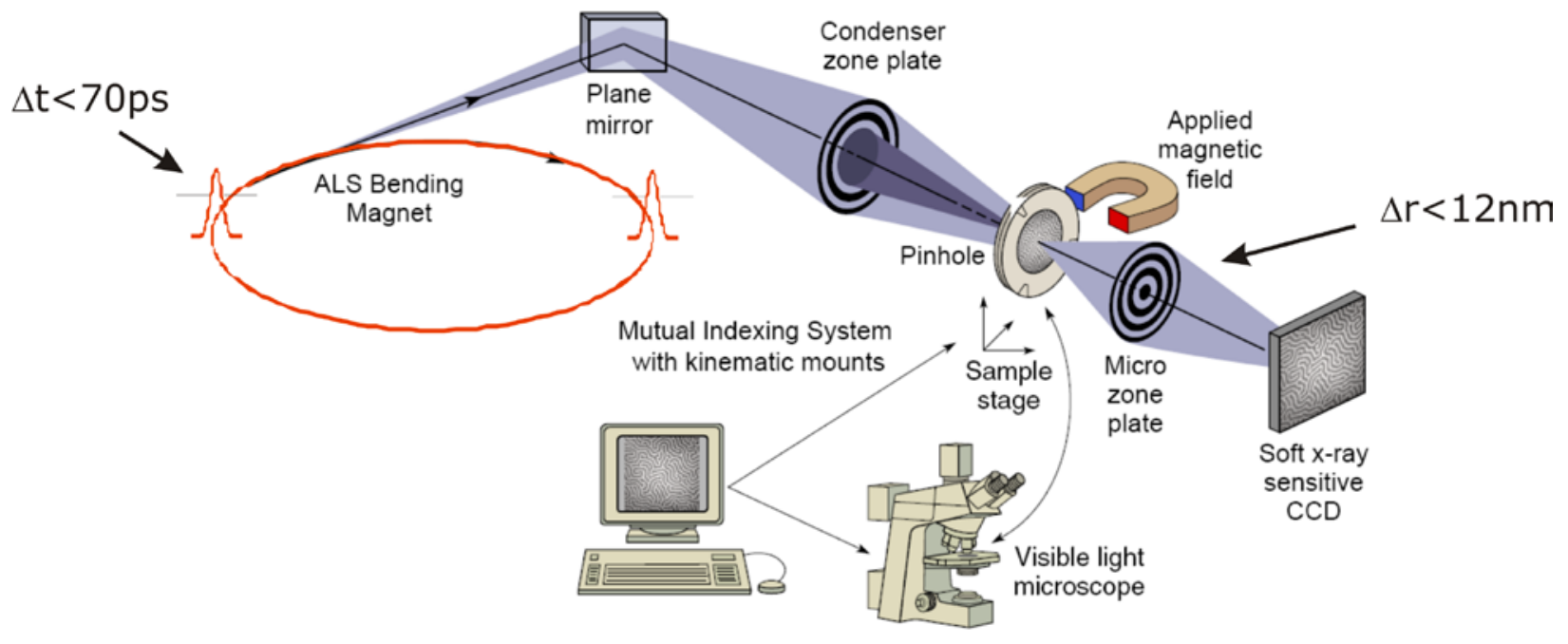

Figure 2 

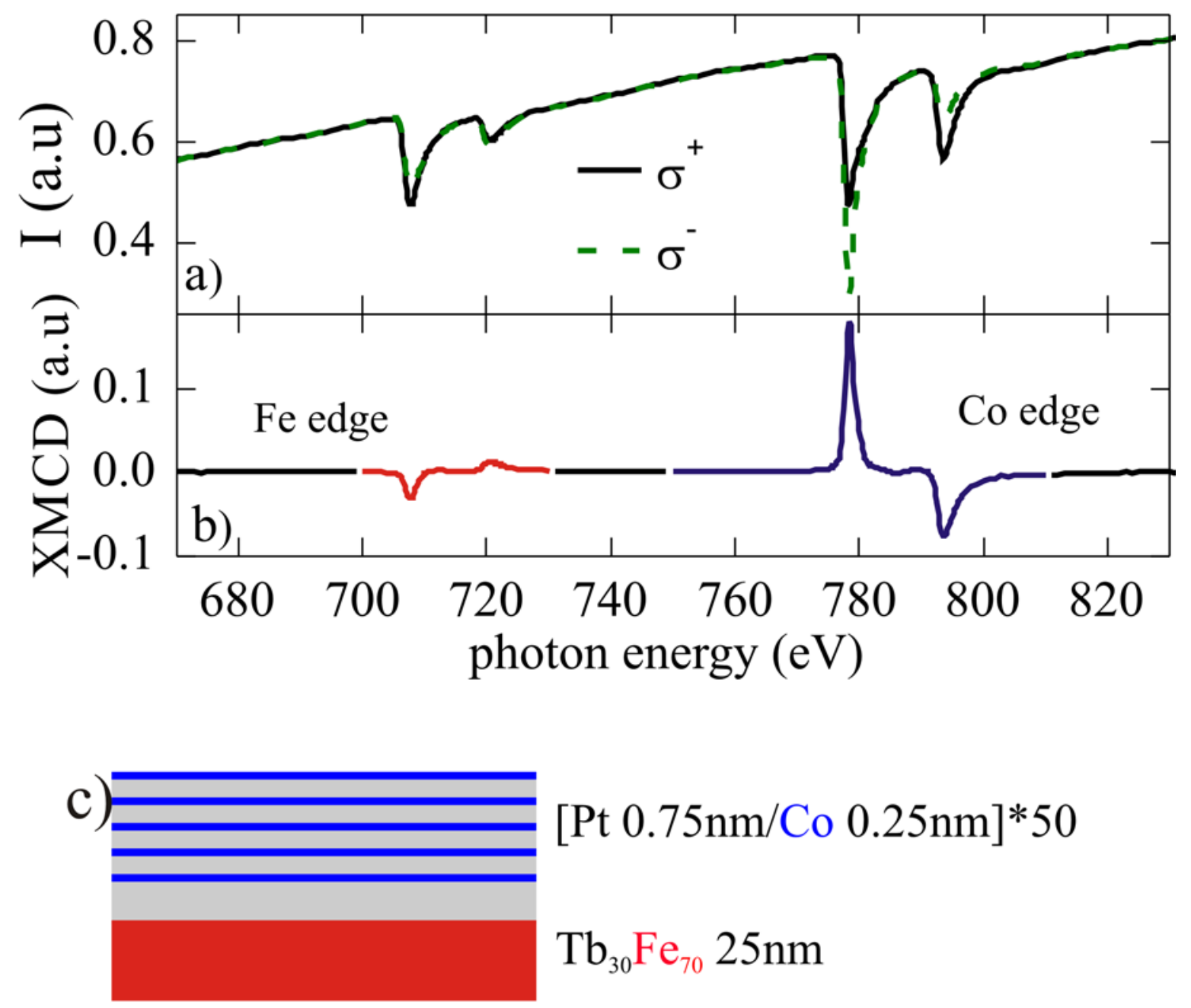

[Pt $0.75 \mathrm{~nm} / \mathrm{Co} 0.25 \mathrm{~nm}] * 50$

$\mathrm{Tb}_{30} \mathrm{Fe}_{70} 25 \mathrm{~nm}$

Figure 3 

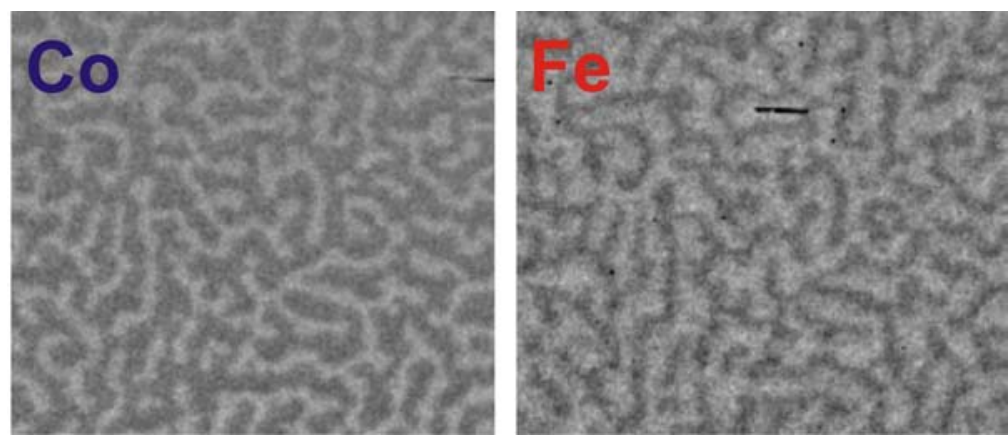

Figure 4 

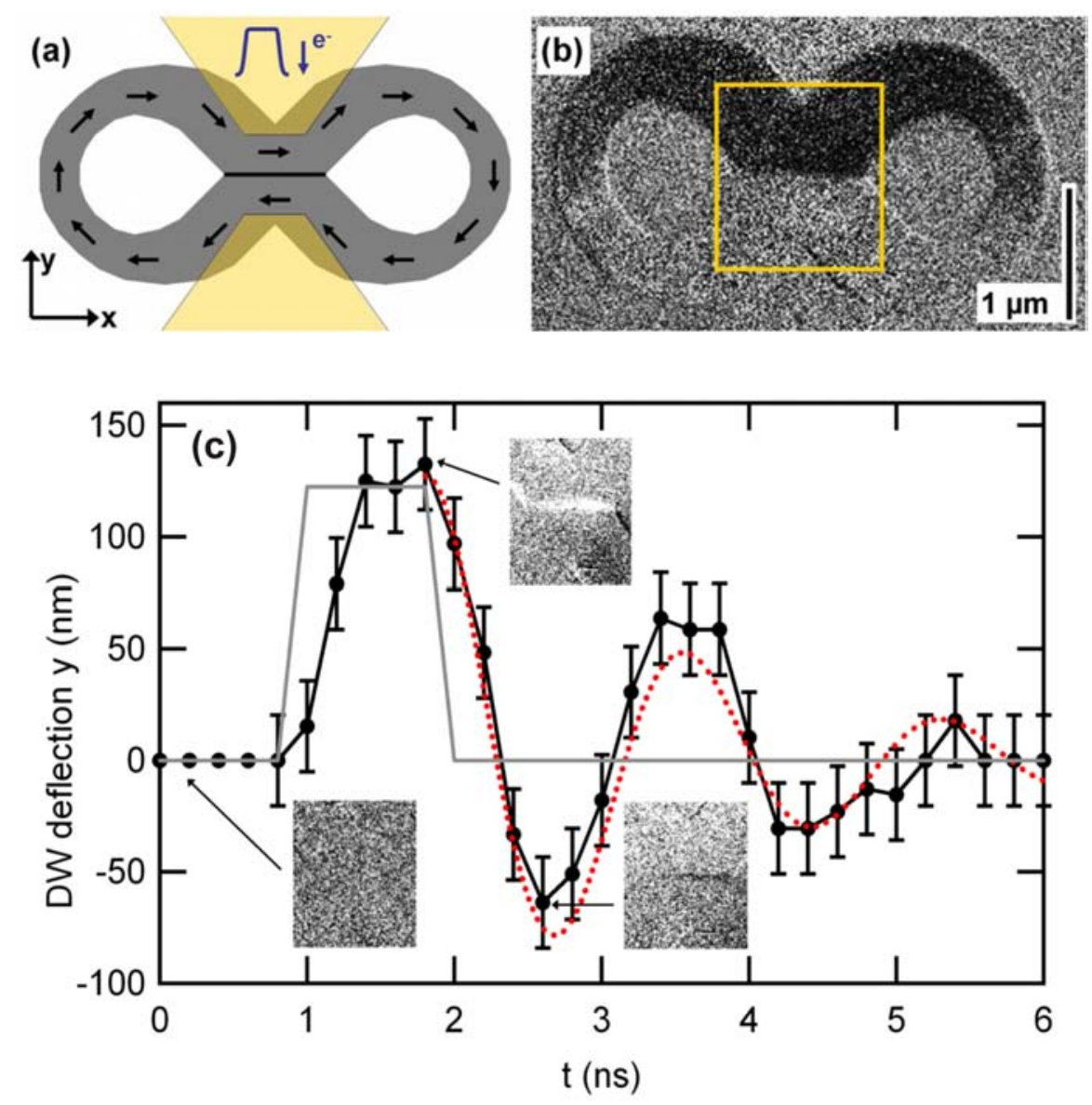

Figure 5 

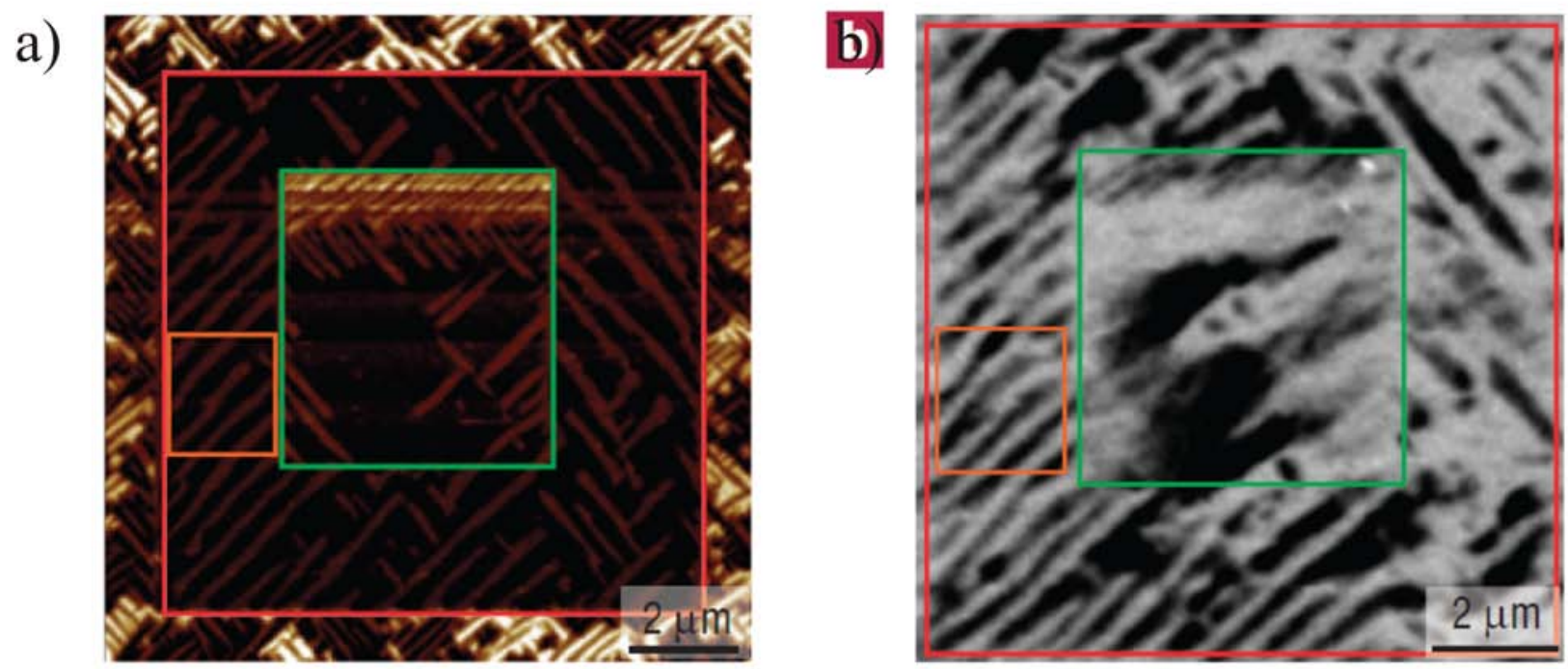

c)
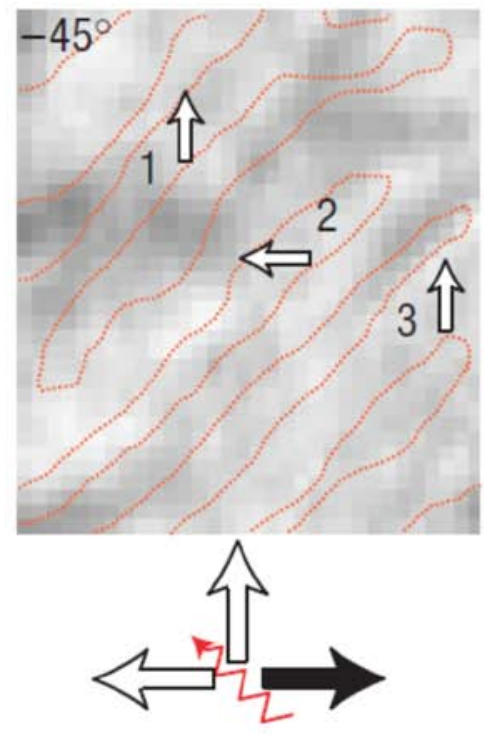
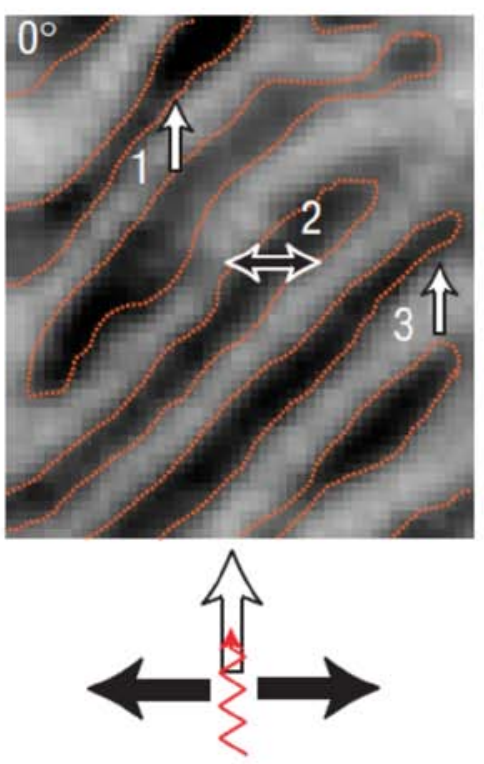
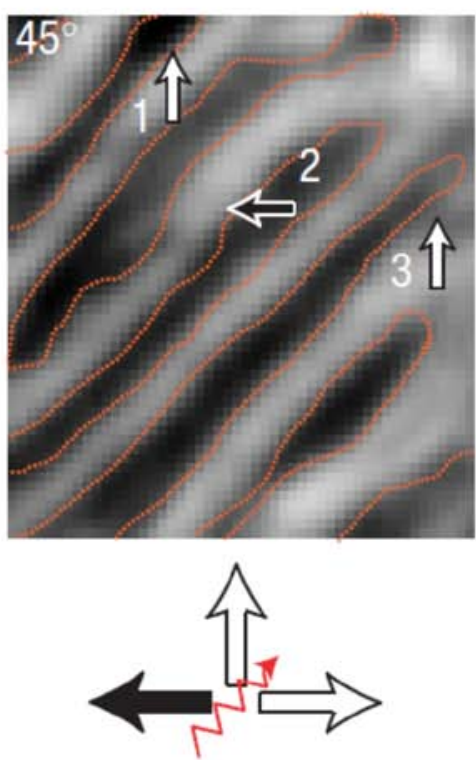

Figure 6 

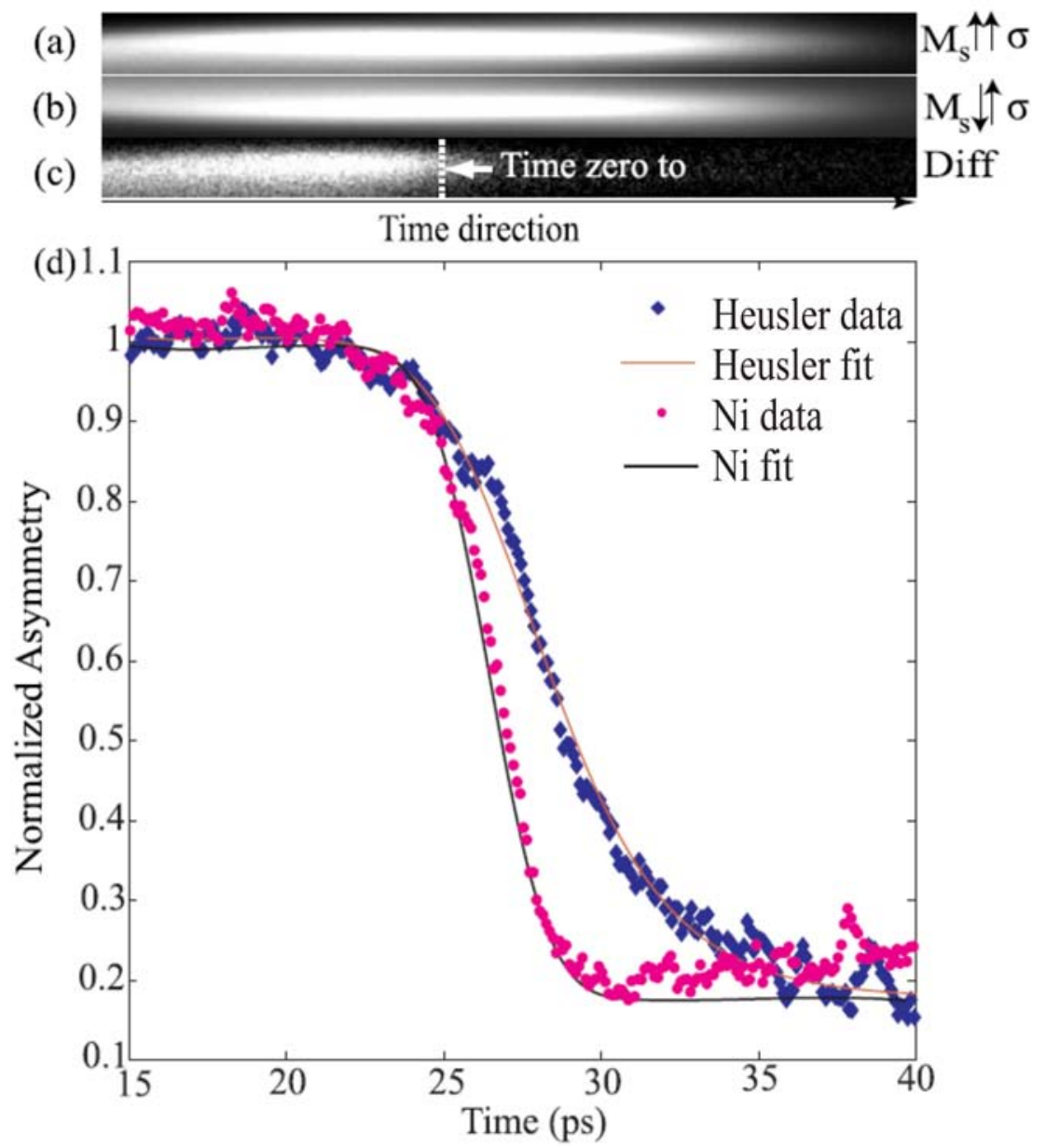

Figure 7 


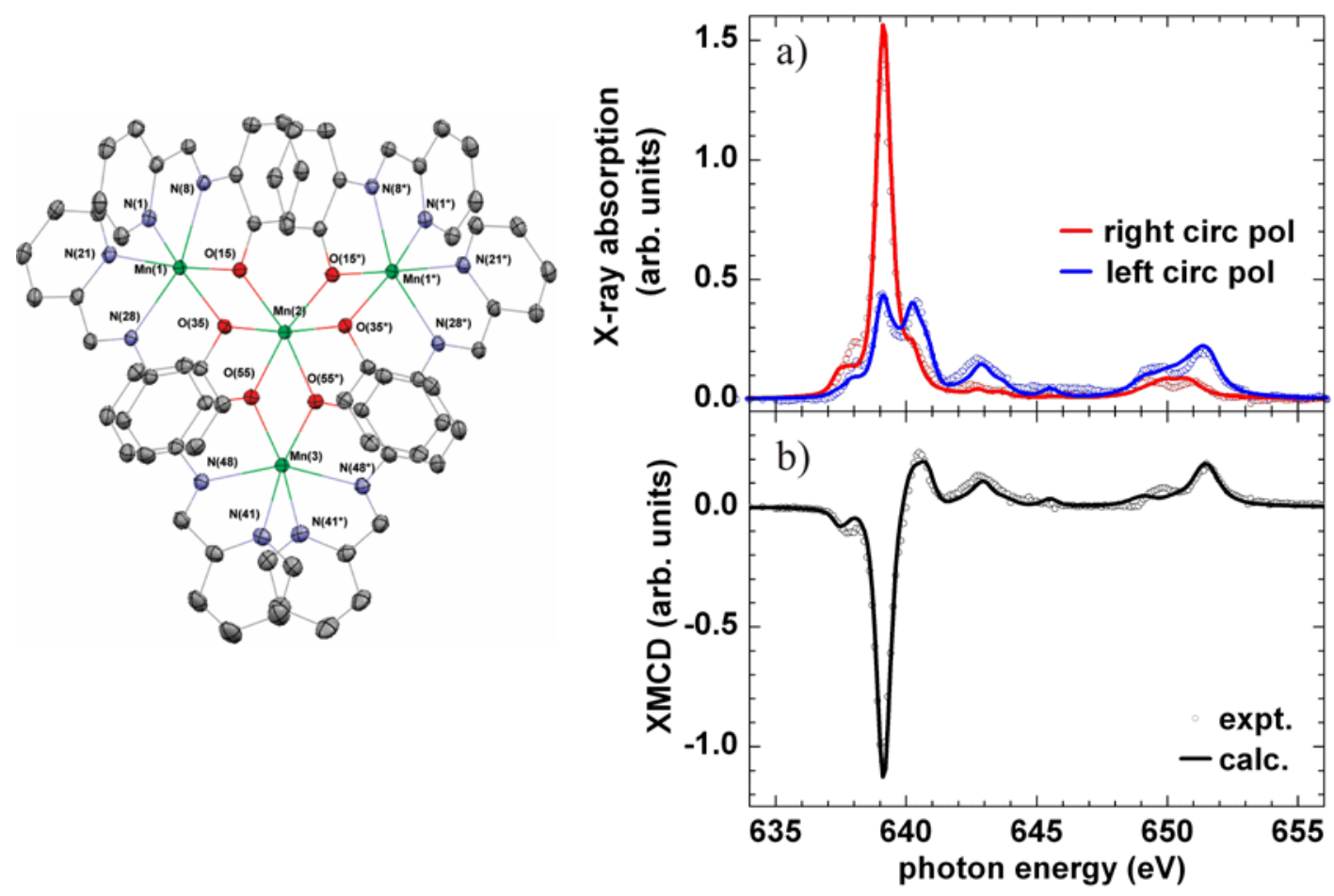

Figure 8 


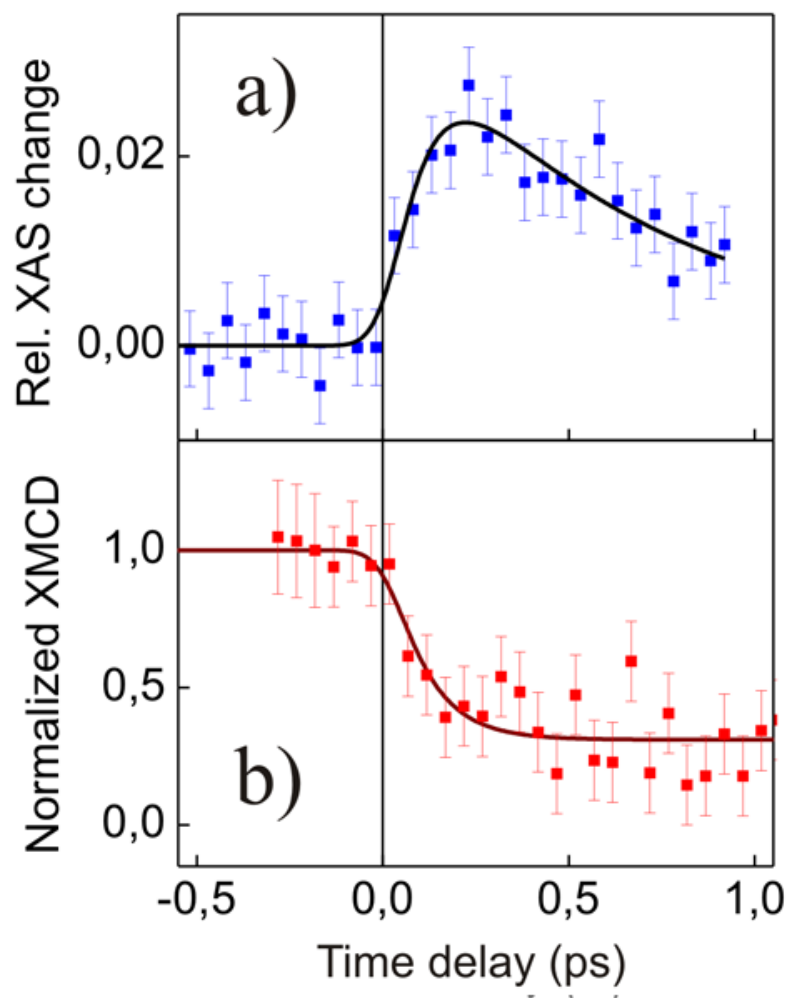

Figure 9 


\section{DISCLAIMER}

This document was prepared as an account of work sponsored by the United States Government. While this document is believed to contain correct information, neither the United States Government nor any agency thereof, nor The Regents of the University of California, nor any of their employees, makes any warranty, express or implied, or assumes any legal responsibility for the accuracy, completeness, or usefulness of any information, apparatus, product, or process disclosed, or represents that its use would not infringe privately owned rights. Reference herein to any specific commercial product, process, or service by its trade name, trademark, manufacturer, or otherwise, does not necessarily constitute or imply its endorsement, recommendation, or favoring by the United States Government or any agency thereof, or The Regents of the University of California. The views and opinions of authors expressed herein do not necessarily state or reflect those of the United States Government or any agency thereof or The Regents of the University of California. 